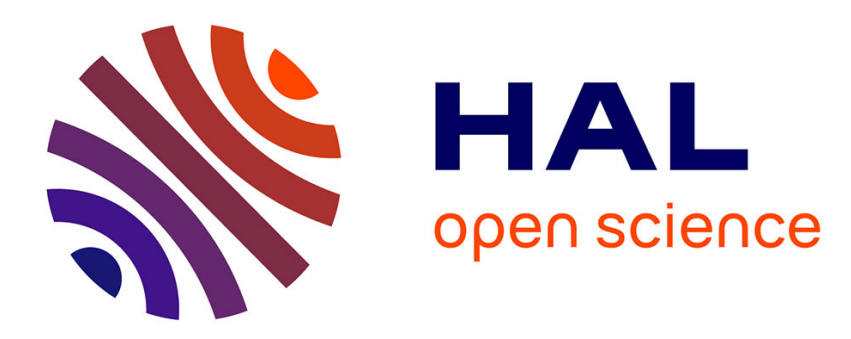

\title{
Appareillage pour l'ionisation thermique des produits de pulvérisation
}

J.-F. Hennequin, M. Couchouron

\section{To cite this version:}

J.-F. Hennequin, M. Couchouron. Appareillage pour l'ionisation thermique des produits de pulvérisation. Revue de Physique Appliquée, 1979, 14 (12), pp.993-1006. 10.1051/rphysap:019790014012099300 . jpa-00244690

\section{HAL Id: jpa-00244690 https://hal.science/jpa-00244690}

Submitted on 1 Jan 1979

HAL is a multi-disciplinary open access archive for the deposit and dissemination of scientific research documents, whether they are published or not. The documents may come from teaching and research institutions in France or abroad, or from public or private research centers.
L'archive ouverte pluridisciplinaire HAL, est destinée au dépôt et à la diffusion de documents scientifiques de niveau recherche, publiés ou non, émanant des établissements d'enseignement et de recherche français ou étrangers, des laboratoires publics ou privés. 


\title{
Appareillage pour l'ionisation thermique des produits de pulvérisation
}

\author{
J.-F. Hennequin et M. Couchouron \\ C.N.R.S., Laboratoire P.M.T.M., Université Paris-Nord, avenue Jean-Baptiste-Clément, 93430 Villetaneuse, France \\ (Reçu le 23 juillet, accepté le 24 septembre 1979)
}

\begin{abstract}
Résumé. - Nous décrivons en détail un appareillage destiné à tester une nouvelle méthode d'analyse élémentaire des solides. Les particules arrachées sous forme neutre au cours du bombardement ionique d'une cible solide viennent interagir avec une surface de tungstène portée à très haute température. Les ions formés sont ensuite analysés dans un spectromètre de masse quadrupolaire. Les résultats ainsi obtenus pour l'ionisation de l'indium après pulvérisation sont similaires à ceux déjà publiés utilisant des jets atomiques thermiques.
\end{abstract}

\begin{abstract}
An apparatus is described in some details for checking a new method of solid elemental analysis. Neutral particles sputtered from a solid target by ion bombardment interact with a tungsten surface heated at very high temperature. The formed ions are then analyzed by means of a quadrupole mass spectrometer. Results for indium ionization after ion sputtering are quite similar to those precedently published where thermal atomic streams were used.
\end{abstract}

1. Introduction. - Lorsque des atomes d'énergie d'ionisation $V$ interagissent avec une surface solide de travail de sortie $W$, une fraction plus ou moins importante de ces atomes se trouve portée à l'état d'ions positifs. C'est ce phénomène, connu depuis 1923, qui constitue l'ionisation thermique (ou ionisation de surface); on en trouvera des revues détaillées en références [1, 2]. Si l'équilibre thermodynamique est réalisé à la température $T$ (température de la surface ionisante), le degré d'ionisation $\alpha$ des particules désorbées est donné par la loi de Saha-Langmuir :

$$
\alpha=\frac{n_{\mathrm{i}}}{n_{0}}=\frac{Q_{\mathrm{i}}}{Q_{0}} \exp \left(-\frac{V-W}{k T}\right)
$$

où $n_{\mathrm{i}}$ et $n_{0}$ sont respectivement les flux de particules désorbées sous forme d'ions ou d'atomes neutres, et où $Q_{\mathrm{i}}$ et $Q_{0}$ sont les fonctions de partition électroniques d'un ion et d'un atome neutre ${ }^{1}$ ).

La surface ionisante (que nous appellerons par la suite : ioniseur) est généralement constituée d'un fil ou d'un ruban d'un métal réfractaire $(\mathrm{Ta}, \mathrm{Re}, \mathrm{W})$ porté à haute température. Dans la plupart des expériences, les atomes dont on étudie l'ionisation sont obtenus par évaporation, à partir d'un four

( ${ }^{1}$ En toute rigueur, la formule (1) suppose négligeables les coefficients de réflexion des ions et des neutres, ce qui semble être usuellement le cas [2]. ou d'un filament chaud; les énergies cinétiques sont thermiques, de l'ordre de $0,25 \mathrm{eV}$ pour une température d'ioniseur de $3000 \mathrm{~K}$. Mais il existe un autre procédé d'atomisation des solides, couramment utilisé pour l'analyse en profondeur ou l'obtention de couches minces : la pulvérisation ionique. Un échantillon du matériau à étudier est alors progressivement érodé par bombardement avec des ions lourds d'une énergie de plusieurs $\mathrm{keV}$. Les particules ainsi éjectées sont principalement des atomes neutres, plus ou moins excités, et les énergies cinétiques peuvent être bien supérieures aux énergies thermiques $[1,3]$. C'est déjà en combinant la pulvérisation ionique et l'ionisation de surface que Bradley [4] a déterminé le rendement de pulvérisation des métaux alcalins.

Comme l'ont remarqué Castaing et al. [5, 6], l'ionisation thermique des produits de pulvérisation devrait permettre d'échapper à deux difficultés majeures que l'on rencontre en spectrométrie de masse des ions secondaires (technique d'analyse S.I.M.S.) : la présence d'ions moléculaires en quantités souvent comparables à celles des ions simples et l'influence marquée de la liaison chimique sur les caractéristiques de l'émission ionique [7]. On peut en effet espérer que l'interaction avec la surface ionisante à haute température provoque à la fois la rupture de la majeure partie des associations moléculaires et l'ionisation selon la loi de Saha-Langmuir, qui ne fait intervenir que des grandeurs atomiques. On disposerait ainsi d'une méthode d'analyse qu'il serait 
facile de rendre quantitative par étalonnage sur des cibles d'éléments purs.

Deux problèmes se posent cependant :

- Un problème théorique : la loi de Saha-Langmuir suppose que l'équilibre thermique est atteint entre la surface ionisante et les atomes adsorbés; en est-il ainsi avec les produits de pulvérisation?

- Un problème pratique de sensibilité : les atomes neutres ne peuvent être focalisés sur l'ioniseur, dont la surface doit donc être aussi grande que le permet l'acceptance angulaire de l'optique de collection des ions formés.

Dans cet article, nous décrivons l'appareillage qui nous a permis de tester cette nouvelle méthode d'analyse et nous évoquons les problèmes que nous avons rencontrés dans sa mise au point. A titre de comparaison, nous étudions l'ionisation thermique d'atomes d'indium, éjectés d'une cible d'indium bombardée par des ions $\mathrm{A}^{+}$de $10 \mathrm{keV}$; les résultats obtenus sont tout à fait comparables à ceux de Wassmuth et Müller [8], qui utilisaient un jet thermique d'atomes d'indium. Quelques travaux préliminaires sur l'analyse d'alliages cuivre-aluminium ont fait l'objet d'une communication antérieure [9].

2. Description de l'appareillage. - Le schéma de principe de l'appareillage est présenté à la figure 1 .

L'échantillon étudié est bombardé sous une incidence de $45^{\circ}$ par des ions $\mathrm{A}^{+}$d'une énergie voisine de $10 \mathrm{keV}$. Ces ions sont produits dans une source à excitation haute fréquence; a rès extraction, ils sont focalisés sur l'échantillon à 1 aide d'une lentille électrostatique. La région bombardée a pour dimensions approximatives : $1 \times 1,5 \mathrm{~mm}^{2}$; elle reçoit

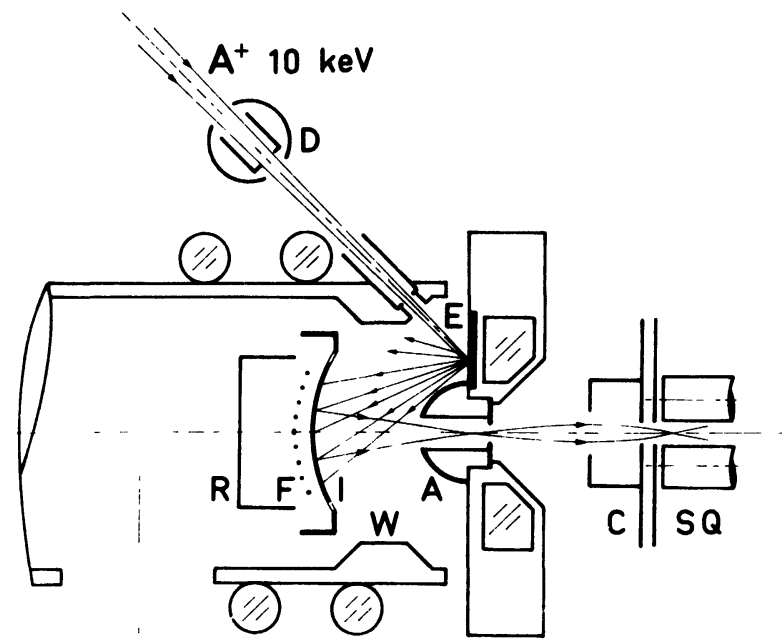

Fig. 1. - Schéma de l'appareil. R : réflecteur ; F : filament ; I : ioniseur ; A : électrode d'accélération ; W : wehnelt ; $\mathrm{E}$ : échantillon-cible ; D : contrôle de courant primaire ; C : cage ; SQ : spectromètre quadrupolaire.

[Experimental set up. $\mathrm{R}$ : reflector ; $\mathrm{F}$ : filament ; I : ionizing surface ; A : acceleration electrode ; W : wehnelt ; C : target ; $\mathrm{D}$ : primary current checking ; $\mathrm{C}$ : cage ; $\mathrm{SQ}$ : quadrupole spectrometer.] un courant d'ions $\mathrm{A}^{+}$d'environ $10 \mu \mathrm{A}$. La mesure de l'intensité de bombardement s'effectue avant expérience en polarisant à $-100 \mathrm{~V}$ l'environnement de la cible, c'est-à-dire l'ioniseur et le wehnelt, de façon à limiter l'effet de l'émission électronique secondaire de la cible. Elle n'est plus possible en cours d'expérience, car le courant cible comprend alors, outre le courant d'ions $\mathrm{A}^{+}$, un courant bien plus important d'électrons en provenance du filament de chauffage de l'ioniseur. Tout au plus conserve-t-on un contrôle de la stabilité des conditions de bombardement, en collectant une fraction du faisceau d'ions incident sur un diaphragme isolé polarisé à $-500 \mathrm{~V}$.

La cible est une pastille métallique de $1 \mathrm{~mm}$ d'épaisseur et de $10 \mathrm{~mm}$ de diamètre ; elle est montée sur une pièce massive en cuivre, refroidie par courant d'eau, qui porte en son centre une électrode hémisphérique en acier inoxydable destinée à l'extraction des ions formés sur l'ioniseur. En cours d'expérience, l'échantillon est inéluctablement soumis au rayonnement de l'ioniseur, mais son échauffement reste limité à une centaine de degrés, comme le prouve la possibilité d'étudier l'indium (température de fusion : $\left.155^{\circ} \mathrm{C}\right)$. Un cache mobile en tantale permet d'éviter le dépôt, à la surface de l'échantillon, des produits de désorption de l'ioniseur pendant sa préparation (voir § 4.2).

L'ioniseur est un ruban de tungstène de $0,1 \mathrm{~mm}$ d'épaisseur et de $14 \mathrm{~mm}$ en largeur, légèrement cintré (rayon de courbure de l'ordre de $20 \mathrm{~mm}$ ), et chauffé par bombardement électronique sur sa face arrière (jusqu'à près de $500 \mathrm{~W}$ pour $3000 \mathrm{~K}$ ). Deux lignes transversales de trous presque tangents fixent la longueur utile à $20 \mathrm{~mm}$ environ et limitent les pertes par conduction thermique. L'ioniseur est monté sur quatre colonnettes en acier inoxydable entretoisées au tiers de leur longueur. L'échauffement des entretoises provoque, par dilatation, l'écartement des extrémités des colonnettes : le rayon de courbure de l'ioniseur demeure ainsi à peu près indépendant de la température, alors que son allongement par dilatation atteint près de $0,4 \mathrm{~mm}$ à $3000 \mathrm{~K}$. Le filament de chauffage est un fil de tungstène de $0,2 \mathrm{~mm}$ de diamètre, de longueur utile $110 \mathrm{~mm}$, disposé en zigzag (en neuf brins de $12 \mathrm{~mm}$ distants de $1,8 \mathrm{~mm}$ ) sur une surface cylindrique parallèle à l'ioniseur, à une distance de l'ordre de $1,5 \mathrm{~mm}$. Un réflecteur en tantale, électriquement relié au côté négatif du filament, protège les supports du rayonnement thermique direct.

Un épais cylindre de cuivre, refroidi par un second circuit d'eau, entoure l'ensemble et, porté au potentiel électrique de l'ioniseur, joue le rôle de wehnelt en focalisant sur le trou de l'électrode d'extraction les ions formés sur l'ioniseur. Le faisceau des ions collectés pénètre ensuite dans un petit spectromètre de masse quadrupolaire dont les caractéristiques sont : rayon du champ : $r_{0}=2,8 \mathrm{~mm}$; longueur des barres : $150 \mathrm{~mm}$; fréquence du champ H.F. : $f=2,13 \mathrm{MHz}$. 
Il s'agit en fait d'un analyseur de gaz résiduel du commerce ; les électrodes de sa source d'ions, convenablement polarisées $(-120 \mathrm{~V}$ sur la cage d'ionisation; autres électrodes à la masse), constituent une optique de transfert qui permet de refocaliser les ions au voisinage de l'entrée du quadrupôle. L'ensemble extraction-spectromètre est mécaniquement centrable par rapport à l'ioniseur.

Le courant ionique est mesuré à l'aide d'un multiplicateur d'électrons à 11 dynodes monté dans l'axe du quadrupôle. Cette disposition nous est imposée par le spectromètre en notre possession; elle n'est pas très heureuse et a pour conséquence un bruit photoélectrique qui devient important lorsque l'ioniseur est à haute température. Un diaphragme de molybdène de $2,4 \mathrm{~mm}$ de diamètre, placé à l'intérieur du canal de l'électrode d'extraction, au voisinage du point de convergence des ions collectés, limite la contamination du quadrupôle par les produits de désorption de l'ioniseur et réduit l'importance du courant photo-électrique parasite, qui peut d'ailleurs, si besoin est, être pratiquement éliminé par une technique de détection synchrone. Il s'agit d'une variante simplifiée de la méthode proposée par Thomas et al. [10] pour améliorer le rapport signal-surbruit d'un spectromètre quadrupolaire identique utilisé en S.I.M.S. Une tension carrée de $\pm 120 \mathrm{~V}$ est appliquée à la cage d'ionisation, par l'intermédiaire d'un transformateur dont la bande passante limitée fixe la fréquence de travail vers $80 \mathrm{~Hz}$, et on mesure l'amplitude crête-à-crête de la modulation du signal de sortie. Pendant l'alternance négative, la transmission des ions par le spectromètre est inchangée ; pendant l'alternance positive, les ions sont refoulés, la transmission devient nulle et le courant mesuré se réduit au seul courant parasite.

La même technique de modulation permet une mesure approchée du gain du multiplicateur, lorsque le courant de l'espèce ionique considérée est suffisamment intense pour qu'on puisse le mesurer directement sur la première dynode. La mesure n'est pas possible en continu parce que la première dynode ne peut être déconnectée du pont de polarisation des dynodes du multiplicateur, dont la résistance totale $(15 \mathrm{M} \Omega)$ est inférieure ou de l'ordre de la résistance d'entrée du picoampèremètre continu en notre possession; l'amplificateur à détection synchrone mesure par contre la tension développée aux bornes d'une résistance de $1 \mathrm{M} \Omega$, constituée par l'impédance d'entrée de l'oscilloscope.

Le pompage principal de l'appareil est assuré par une pompe à diffusion d'huile de $600 \mathrm{l} / \mathrm{s}$ surmontée d'un piège à azote liquide. La partie source d'ions dispose d'un pompage auxiliaire d'environ $50 \mathrm{l} / \mathrm{s}$ par une autre petite pompe à diffusion; elle est séparée de la chambre d'expériences par un trou de $8 \mathrm{~mm}$ de diamètre qui peut être fermé par un clapet d'isolement. On peut ainsi, non seulement procéder à des interventions sur une partie de l'appareillage en laissant sous vide l'autre partie, mais aussi interrompre le bombardement ionique en cours d'expérience, sans toucher aux autres réglages. Ceci est précieux pour mettre en évidence un signal parasite (dû à la réévaporation de dépôts antérieurs sur les parois de l'appareil ou à la désorption d'impuretés de l'ioniseur), signal qu'il convient évidemment de défalquer du signal observé avec bombardement pour obtenir la seule contribution des produits de pulvérisation.

Le vide limite dans l'appareil atteint $2 \times 10^{-7}$ torr, mais la pression remonte vers $10^{-6}$ torr pendant le bombardement ionique. L'analyse du gaz résiduel montre qu'outre l'argon, celui-ci est essentiellement constitué de vapeur d'eau provenant vraisemblablement pour une large part de microfuites dans les circuits de refroidissement.

3. Collection des ions. - 3.1 ExPRESSION DU COURANT COLLECTÉ. - L'intensité du courant d'ions primaires étant noté $I_{1}(\sim 10 \mu \mathrm{A})$, l'échantillon-cible reçoit $I_{1} / q$ ions primaires monochargés par seconde ( $q$ est la charge électrique élémentaire) et perd dans le même temps $S I_{1} / q$ atomes, $S$ désignant le rendement de pulvérisation du matériau. Pour les métaux usuels bombardés à $45^{\circ}$ par des ions $\mathrm{A}^{+}$de $10 \mathrm{keV}$, les valeurs de $S$ se situent entre 2 et 20 atomes/ion [1, 3]. La répartition angulaire des produits de pulvérisation suit sensiblement la loi d'émission en cosinus à ces énergies de bombardement, si la cible est polycristalline (étant donné la taille de la région bombardée, ceci suppose des microcristaux de dimensions très inférieures au millimètre). Comme le sommet de l'ioniseur est situé à $l=22 \mathrm{~mm}$ de la région bombardée, dans une direction dirigée à $30^{\circ}$ de la normale à la surface de la cible, et que la normale au sommet de l'ioniseur fait elle-même un angle de $30^{\circ}$ avec la direction moyenne des particules incidentes, le flux $N$ des atomes incidents, au voisinage du sommet de l'ioniseur, vaut :

$N=\frac{S I_{1}}{q} \times \frac{\cos ^{2} 30^{\circ}}{\pi l^{2}} \simeq 5 \times 10^{-4} \frac{S I_{1}}{q}$ atomes $/ \mathrm{mm}^{2} \mathrm{~s}$,

étant entendu que certains de ces atomes peuvent être ionisés et/ou associés en molécules. Le champ électrique d'accélération des ions désorbés constitue en fait un champ retardateur pour les ions secondaires émis par l'échantillon, dont la majeure partie n'atteint pas l'ioniseur. Ceci n'est guère gênant avec les métaux purs, dont le rendement ionique est très inférieur à l'unité [7], mais pourrait le devenir avec certains composés (oxydes, halogénures), où l'émission à l'état d'ions semble prédominante.

Le flux $N$ est de l'ordre de $10^{11}$ atomes $/ \mathrm{mm}^{2} \mathrm{~s}$, ce qui constitue une valeur élevée par rapport aux jets thermiques $[2,8,11]$. La concentration superficielle des particules adsorbées à la surface de l'ioniseur se fixe en régime permanent à la valeur $N \tau$, où $\tau$ est 
le temps moyen de séjour à la température considérée. Pour que le travail de sortie de l'ioniseur n'en soit pas affecté, il faut que sa température soit assez élevée pour que : $N \tau \ll 10^{13} \mathrm{at} / \mathrm{mm}^{2}$, valeur limite qui correspond à la concentration superficielle des atomes de tungstène. Un problème similaire se pose à propos de l'oxydation de l'ioniseur par l'oxygène ou par l'eau du gaz résiduel.

En régime permanent, le flux $\left(n_{\mathrm{i}}+n_{0}\right)$ des particules désorbées est égal à $N$. Admettant la thermalisation de ces particules avec l'ioniseur, au moins pour les états électroniques, on a : $n_{\mathrm{i}}=\alpha n_{0}$, où $\alpha$ est donné par la formule (1), et le flux d'ions désorbés s'écrit :

$$
n_{\mathrm{i}}=\beta N=5 \times 10^{-4} \beta \frac{S I_{1}}{q} \text { ions } / \mathrm{mm}^{2} \mathrm{~s}
$$

en introduisant le coefficient d'ionisation $\beta$, c'est-à-dire le rapport :

$$
\beta=\frac{n_{\mathrm{i}}}{n_{\mathrm{i}}+n_{0}}=\frac{\alpha}{1+\alpha}
$$

qui diffère très peu de $\alpha$ pour $\alpha \ll 1$, et tend vers 1 pour $\alpha \gg 1$.

Une partie seulement des ions formés sur l'ioniseur contribue au courant collecté. Introduisons l'aire théorique intégralement imagée $s$ comme la surface de l'ioniseur d'où proviendraient les ions effectivement collectés par le système d'extraction s'ils étaient intégralement transmis par l'optique de transfert et par le quadrupôle, quelles que soient leur énergie initiale $\varepsilon$ et leur direction d'émission $\varphi$. Le courant d'ions arrivant sur la première dynode du multiplicateur d'électrons vaut alors : $5 \times 10^{-4} \beta s S I_{1}$, et, si $G$ est le gain du multiplicateur pour l'espèce ionique collectée, le courant à la sortie vaut :

$$
I=5 \times 10^{-4} \beta s G S I_{1}
$$

où le paramètre $s$ reste à déterminer.

Dans le cas d'un alliage ou d'un composé, le courant $I\left(A^{+}\right)$correspondant au constituant $A$ de concentration atomique $c_{\mathrm{A}}$ est donné par la formule similaire :

$$
I\left(A^{+}\right)=5 \times 10^{-4} \beta s G c_{\mathrm{A}} S I_{1}
$$

où $\beta, s$ et $G$ dépendent de $\mathrm{A}$ et où le rendement de pulvérisation $S$ de l'alliage est généralement lui-même fonction de $c_{\mathrm{A}}$. En régime permanent, les flux de particules éjectées par pulvérisation sont en effet nécessairement proportionnels aux concentrations des constituants correspondants dans la masse de l'échantillon $[5,6]$.

3.2 L'AIRE THÉORIQUE INTÉGRALEMENT IMAGÉE. Les propriétés optiques du système d'extraction des ions et de l'optique de transfert font l'objet d'une étude détaillée en annexe 1 . Ces calculs montrent que c'est en fait l'acceptance du quadrupôle qui détermine l'aire théorique intégralement imagée $s$. Les calculs théoriques [12] et les résultats expérimentaux [13] font apparaître que cette acceptance est fixée, pour une résolution $R=M / \Delta M$ donnée, par le nombre $n$ de cycles H.F. subis par l'ion sur une longueur $r_{0}$, soit :

$$
n=f r_{0} \sqrt{\frac{m}{2 q V_{\mathrm{A}}}}
$$

où $m$ est la masse de l'ion (et $M$ sa masse molaire) et $q V_{\mathrm{A}}$ son énergie, laquelle est définie par la tension $V_{\mathrm{A}}$ appliquée à l'ioniseur puisque le potentiel de l'axe du quadrupôle est celui de la masse. Les conditions d'attaque optimales d'un quadrupôle correspondent à $n \simeq 1$ cycle H.F. $/ r_{0}$ ou un peu plus, pour un faisceau d'ions convergent en un cross-over (région d'amincissement) situé légèrement à l'intérieur du champ quadrupolaire [12]

Plus précisément, dans chacune des directions $u$ $\left(x\right.$ ou $y$ ) $\left({ }^{2}\right)$, la transmission des ions n'atteint plus que $50 \%$ pour un angle limite d'injection $\theta_{50}^{u}$, à condition que le rayon du cross-over soit inférieur à une valeur maximale $u_{\mathrm{m}}$ [14]. Ecrivant alors la relation de Lagrange-Helmholtz pour un ion émis avec l'énergie $\varepsilon\left(\varepsilon \simeq k T \ll q V_{\mathrm{A}}\right)$ presque perpendiculairement à l'axe optique $(\varphi \simeq \pi / 2)$, on obtient le rayon $r_{u}$ de l'aire théorique intégralement imagée

$$
\sqrt{\varepsilon} r_{u} \sin \varphi=\sqrt{q V_{\mathrm{A}}} u_{\mathrm{m}} \theta_{50}^{u} .
$$

Tenant compte des deux directions $x$ et $y$, et supposant $s$ de forme elliptique, on en déduit :

$$
s=\pi r_{x} r_{y}=\pi \frac{q V_{\mathrm{A}}}{k T} x_{\mathrm{m}} y_{\mathrm{m}} \theta_{50}^{x} \theta_{50}^{y}
$$

que l'on peut aussi écrire, en introduisant $n$ à la place de $V_{\mathrm{A}}$ :

$$
s=\frac{\pi}{2} \frac{m}{k T}\left(\frac{f r_{0}}{n}\right)^{2} x_{\mathrm{m}} y_{\mathrm{m}} \theta_{50}^{x} \theta_{50}^{y}
$$

faisant ainsi apparaître que $s$ dépend, non seulement des caractéristiques du quadrupôle, mais aussi de la nature des ions observés, de leur énergie après accélération et de la température de l'ioniseur.

$\mathrm{Si}$, comme le confirme l'évaluation de $s$ effectuée en annexe 2, c'est bien le quadrupôle qui limite l'aire imagée, $s$ varie en $1 / T$ et par conséquent le coefficient d'ionisation $\beta$ est proportionnel à $T I$, alors qu'il est proportionnel à $I$ lorsque l'aire imagée est limitée par la taille de la source émissive. On pourrait envisager, pour accroître $s$ et donc le courant collecté $I$, d'abaisser $n$ en augmentant $V_{\mathrm{A}}$. En fait, $u_{\mathrm{m}}$ et $\theta_{50}^{u}$

$\left({ }^{2}\right)$ Dans un quadrupôle, la direction $x$ est la direction perpendiculaire à l'axe définie par les deux barres opposées qui reçoivent la tension continue de même signe que la charge des ions ; la direction $y$ est celle définie par les deux barres portées à la tension continue de signe contraire à la charge des ions. 
diminuent rapidement pour $n<1$, et il n'y a aucun avantage à descendre notablement au-dessous de 1 cycle H.F./ $r_{0}$. Seul le montage d'un quadrupôle de plus grande taille permettrait d'augmenter quelque peu la valeur de $s\left(u_{\mathrm{m}}\right.$ étant proportionnel à $r_{0}, s$ varie en $r_{0}^{4}$ si la fréquence $f$ est maintenue constante et si on agit sur $V_{\mathrm{A}}$ pour garder $n$ constant).

4. Mise au point du montage. - 4.1 Chauffage DE L'IONISEUR. - L'ioniseur est chauffé par bombardement électronique sur sa face arrière; la figure 2 précise le schéma électrique du montage. Bien que de mise en œuvre plus délicate que l'effet Joule, ce mode de chauffage a été adopté parce que les intensités des courants $I_{\mathrm{e}}$ et $I_{\mathrm{f}}$ n'excèdent pas quelques ampères et sont donc aisément manipulables, et surtout parce que l'ioniseur est équipotentiel, ainsi que nous l'avons implicitement admis au paragraphe 3.2.

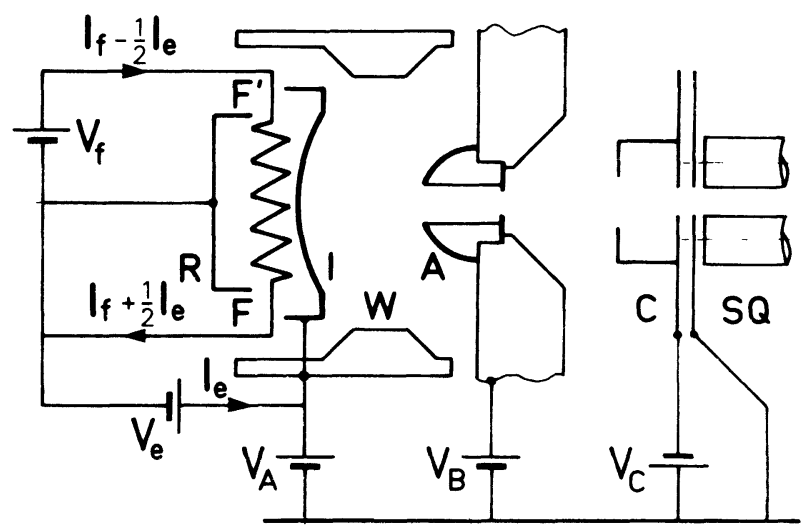

Fig. 2. - Schéma électrique du montage (usuellement $: V_{\mathrm{f}} \simeq 16 \mathrm{~V}$; $I_{\mathrm{f}}=4,8 \mathrm{~A} ; V_{\mathrm{e}}: 0$ à $600 \mathrm{~V} ; I_{\mathrm{e}}: 0$ à $1 \mathrm{~A} ; V_{\mathrm{A}}=20 \mathrm{~V} ; V_{\mathrm{B}}=0 ;$ $\left.V_{\mathrm{C}}=-120 \mathrm{~V}\right)$.

[Electric scheme (typical values : $V_{\mathrm{f}} \simeq 16 \mathrm{~V} ; I_{\mathrm{f}}=4,8 \mathrm{~A} ; V_{\mathrm{e}}: 0$ to $600 \mathrm{~V} ; I_{\mathrm{e}}: 0$ to $\left.1 \mathrm{~A} ; V_{\mathrm{A}}=20 \mathrm{~V} ; V_{\mathrm{B}}=0 ; V_{\mathrm{C}}=-120 \mathrm{~V}\right)$.]

Le chauffage par effet Joule nécessiterait par contre un courant de plusieurs centaines d'ampères, d'où il résulterait un champ magnétique transversal d'une centaine de gauss au voisinage du ruban ioniseur et une chute de tension de quelques volts d'une extrémité à l'autre du ruban, nullement négligeable devant la tension $V_{\mathrm{A}}-V_{\mathrm{B}}$ d'accélération des ions. Ces deux effets conduiraient à une déviation supplémentaire des trajectoires ioniques et la position de l'aire imagée deviendrait fonction de la température de l'ioniseur !

Si le courant $I_{\mathrm{f}}$ dans le filament de chauffage est suffisamment élevé, le courant thermo-électronique $I_{\mathrm{e}}$ n'est plus limité par l'émissivité électronique du tungstène (fonction rapidement croissante de la température), mais uniquement par la charge d'espace. Dans le cas d'une cathode émissive plane de surface $A$ disposée parallèlement et à la distance $e$ de l'anode, l'intensité $I_{\mathrm{e}}$ varie alors avec la tension $V_{\mathrm{ak}}$ appliquée entre anode et cathode selon la loi bien connue :

$$
I_{\mathrm{e}}=\frac{4}{9} \varepsilon_{0} \sqrt{\frac{2 q}{\mu}} \frac{A}{e^{2}} V_{\mathrm{ak}}^{3 / 2}
$$

où $q$ et $\mu$ sont respectivement la charge et la masse de l'électron $\left(\varepsilon_{0}=8,85 \times 10^{-12} \mathrm{~F} / \mathrm{m}\right)$. Comme le montre la figure 3, la relation $I_{\mathrm{e}} \propto V_{\mathrm{ak}}^{3 / 2}$ n'est vérifiée dans notre montage qu'aux faibles valeurs de $V_{\mathrm{e}}$, même en prenant $V_{\mathrm{ak}}=V_{\mathrm{e}}-\frac{1}{2} V_{\mathrm{f}}$ pour tenir approximativement compte de la chute de tension le long du filament.

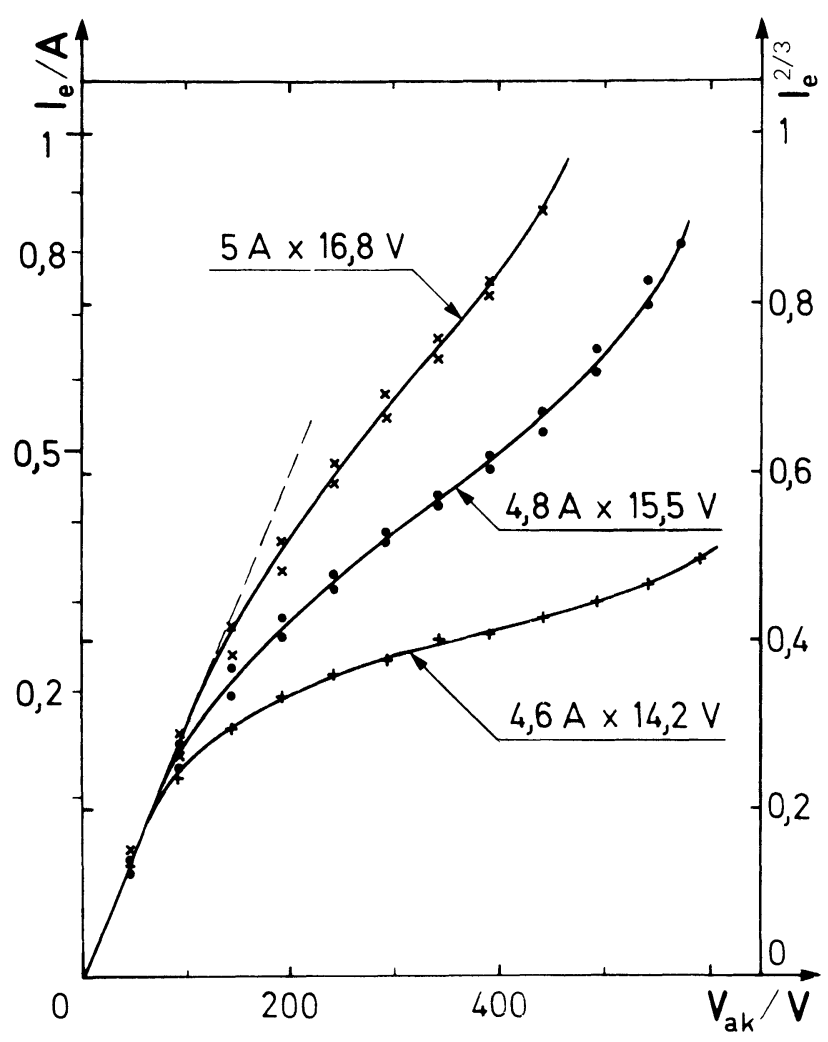

Fig. 3. - Caractéristique courant-tension de chauffage de l'ioniseur $\left(V_{\mathrm{ak}}=V_{\mathrm{e}}-\frac{1}{2} V_{\mathrm{f}}\right)$ pour différentes valeurs du courant de filament.

[Current-voltage characteristic for the heating of the ionizing surface $\left(V_{\mathrm{ak}}=V_{\mathrm{e}}-\frac{1}{2} V_{\mathrm{f}}\right)$ for different values of the filament current.]

Deux raisons peuvent expliquer ce comportement :

- L'une des moitiés du filament, parcourue par un courant inférieur à $I_{\mathrm{f}}$ (jusqu'à $I_{\mathrm{f}}-\frac{1}{2} I_{\mathrm{e}}$ à l'extrémité), est moins chauffée que l'autre. Lorsque $I_{e}$ n'est plus négligeable devant $I_{\mathrm{f}}$, une partie du filament peut ne plus travailler en régime de charge d'espace et son émission se sature. Ce phénomène se produit naturellement d'autant plus tôt que $I_{\mathrm{f}}$ est plus faible ; il semble paradoxalement s'atténuer aux fortes valeurs de $I_{\mathrm{e}}\left(V_{\mathrm{e}}>400 \mathrm{~V}\right)$, où le rayonnement thermique de l'ioniseur devient important et peut contribuer à relever très appréciablement la température du fila- 
ment (au risque d'en provoquer la fusion à l'autre extrémité, parcourue par $I_{\mathrm{f}}+\frac{1}{2} I_{\mathrm{e}}$ ).

- La distance moyenne $e$ entre filament et ioniseur varie avec la température. Aux faibles valeurs de $I_{\mathrm{e}}$, où la totalité de la surface du filament $\left(A \simeq 70 \mathrm{~mm}^{2}\right)$ émet en régime de charge d'espace, la formule (4) conduit à $e \simeq 1 \mathrm{~mm}$, en bon accord avec la valeur prévue à la construction (d'autant qu'avec d'autres ioniseurs, nous avons trouvé jusqu'à $1,3 \mathrm{~mm}$, en raison de légères variations de la longueur des ioniseurs lors de leur fabrication). C'est au cours des régimes transitoires que se manifeste clairement le fait que $e$ n'est pas constant : une modification de $V_{\mathrm{e}}$ s'accompagne d'une variation presque instantanée de $I_{\mathrm{e}}$, suivie d'une évolution plus lente, sur un temps de l'ordre de quelques minutes, qui correspond à la dilatation des entretoises.

En toute rigueur, la différence de potentiel entre filament et ioniseur varie le long du ruban ioniseur de $V_{\mathrm{e}}$ à $V_{\mathrm{e}}-V_{\mathrm{f}}$ et la puissance électrique reçue par unité de surface d'ioniseur n'est pas uniformément répartie. Admettant la loi de Stefan pour déterminer la température locale de l'ioniseur, on montre aisément qu'en régime de charge d'espace, la variation $\Delta T$ de température qui en résulte, d'une extrémité à l'autre du ruban ioniseur, est donnée par :

$$
\frac{\Delta T}{T} \simeq \frac{5}{8} \frac{V_{\mathrm{f}}}{V_{\mathrm{e}}}
$$

ce qui conduit au pire à $\Delta T=50 \mathrm{~K}$ sur $16 \mathrm{~mm}$, valeur négligeable devant les fluctuations de température dues aux variations locales de la distance filament-ioniseur (voir $§ 4.2$ ).

On pourrait envisager d'effectuer le chauffage en régime de saturation, en diminuant un peu $I_{\mathrm{f}}$ et en utilisant une tension $V_{\mathrm{e}}$ nettement plus élevée. Le courant électronique $I_{\mathrm{e}}$ serait fixé par la loi de Richardson-Dushman :

$$
I_{\mathrm{e}} \propto T_{\mathrm{f}}^{2} \exp \left(-\frac{q W}{k T_{\mathrm{f}}}\right) ;
$$

il serait fonction de la température $T_{\mathrm{f}}$ du filament, mais indépendant de $e$ et donc des déformations mutuelles du filament et de l'ioniseur. Malheureusement, $T_{\mathrm{f}}$ varie quelque peu le long du filament, car le courant électronique $I_{\mathrm{e}}$ n'est pas négligeable devant $I_{\mathrm{f}}$. A la variation d'émissivité locale qui en résulte, il correspondrait une variation $\Delta T$ de la température le long du ruban ioniseur telle que :

$$
\frac{\Delta T}{T} \simeq \frac{1}{4}\left(1+\frac{q W}{k T_{\mathrm{f}}}\right) \frac{I_{\mathrm{e}}}{I_{\mathrm{f}}}
$$

ce qui, pour $T_{\mathrm{f}}=3000 \mathrm{~K}$, conduirait à $\Delta T / T \simeq 1$. C'est là une valeur inacceptable, même si ce calcul grossier a négligé la conduction thermique, tant dans le filament que dans l'ioniseur, qui tendrait à diminuer la valeur de $\Delta T$.
De plus, la température du filament est fixée pour une part par le rayonnement qu'il reçoit de l'ioniseur. Cette contribution, que nous avons négligée dans le calcul précédent, est d'autant plus importante que la température de l'ioniseur est plus élevée. Il apparaît ainsi un risque d'emballement thermique lors $\mathrm{du}$ fonctionnement en régime de saturation, qui se traduit au mieux par le retour au régime de charge d'espace, au pire par la rupture prématurée du filament ou le perçage de l'ioniseur. Ce genre d'incidents nous est arrivé assez fréquemment pour montrer que le risque n'est pas illusoire ! Pour la même raison, il est impossible d'utiliser une cathode à oxydes à la place du filament de tungstène.

Le chauffage en régime de charge d'espace est donc le seul mode de fonctionnement acceptable. Il est prudent de stabiliser la tension $V_{\mathrm{f}}$ aux bornes du filament et de limiter l'intensité $I_{\mathrm{e}}$ de l'alimentation qui fournit $V_{\mathrm{e}}$ pour éviter tout emballement lors d'une montée en température trop rapide.

4. 2 ETAlonNAge EN TEMPÉRATURE DE L'IONISEUR. L'étalonnage en température de l'ioniseur est effectué au pyromètre optique à disparition de filament, en remplaçant le spectromètre quadrupolaire par un hublot. En interposant sur le faisceau lumineux un second hublot identique, nous avons pu évaluer à $1 \%$ environ la correction à apporter à la température mesurée pour tenir compte du facteur de transmission du hublot. Le diaphragme monté dans l'électrode d'extraction est retiré pour faciliter la visée et surtout pour que seule la pupille d'entrée du pyromètre vienne limiter l'ouverture du faisceau. Le pointé s'effectue sur une surface de l'ordre du $\mathrm{mm}^{2}$; la précision sur la mesure de la température est de l'ordre de $\pm 20 \mathrm{~K}$, l'incertitude provenant tout autant de l'appréciation de la disparition du filament que de la méconnaissance de la valeur exacte de l'émissivité du tungstène.

Avant toute mesure, l'ioniseur neuf est lentement monté à $2800 \mathrm{~K}$ pour éliminer les impuretés carbonées, puis recuit pendant plusieurs heures vers $2300 \mathrm{~K}$ aux alentours de $2 \times 10^{-6}$ torr. Ce traitement de vieillissement a un double but : il libère les contraintes mécaniques internes du tungstène (et il n'est pas rare que les déformations qui en résultent rendent l'ioniseur inutilisable...) et provoque un réarrangement cristallographique de la surface [15], par formation de faces vicinales (100). Le travail de sortie du tungstène $\left({ }^{3}\right)$ se fixe ainsi à la valeur $W=4,60 \mathrm{eV}$, lorsque la température est assez élevée pour que la contamination par l'oxygène du gaz résiduel soit négligeable $[8,10,16]$.

La figure 4 reproduit la courbe d'étalonnage en température de l'ioniseur pour différentes valeurs du

$\left({ }^{3}\right)$ Dans ces conditions, il n'est pas nécessaire de distinguer le travail de sortie apparent des électrons de celui des ions, lequel est un peu plus élevé pour une surface polycristalline $[2,17]$. 


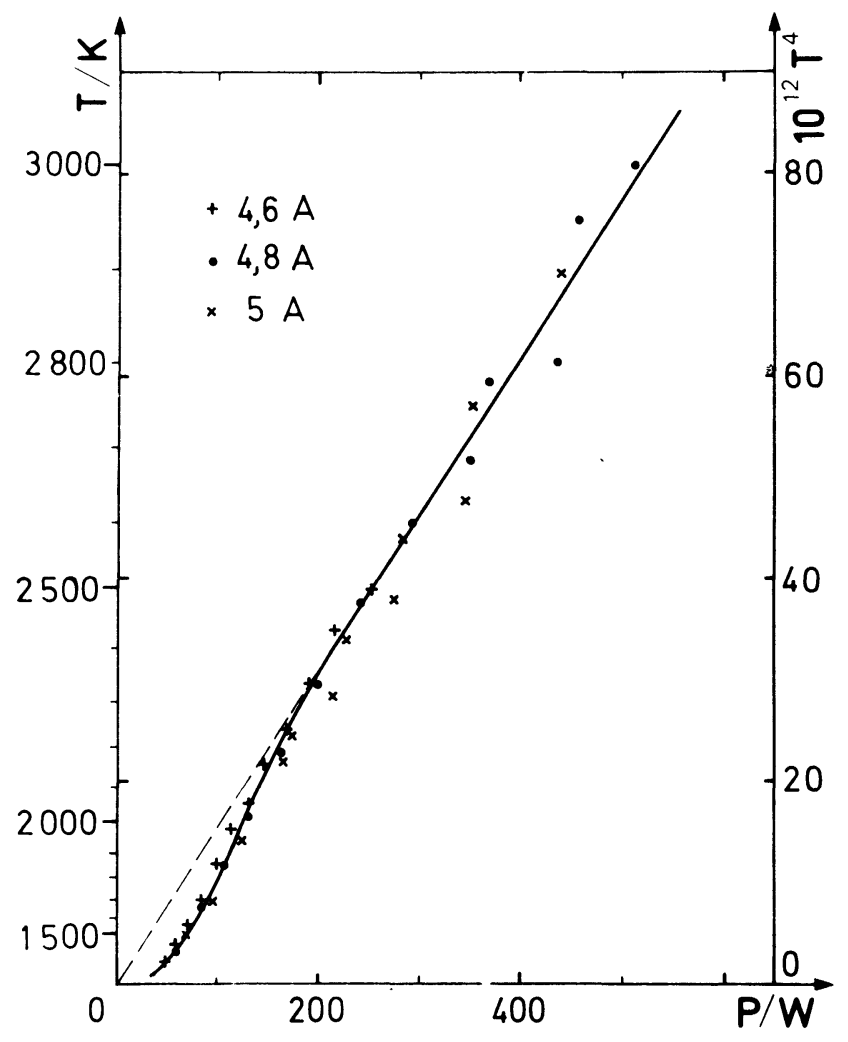

Fig. 4. - Etalonnage en température de l'ioniseur pour différentes valeurs du courant de filament $\left(P=V_{\mathrm{ak}} I_{\mathrm{e}}+0,7 V_{\mathrm{f}} I_{\mathrm{f}}\right)$.

[Temperature of the ionizing surface versus the heating power : $P=V_{\mathrm{ak}} I_{\mathrm{e}}+0,7 V_{\mathrm{f}} I_{\mathrm{f}}$, for different values of the filament current.]

courant de chauffage $I_{\mathrm{f}}$. La puissance de chauffage $P$ comprend la puissance $P_{\mathrm{e}}=V_{\text {ak }} I_{\mathrm{e}}$ du bombardement électronique (terme prépondérant à haute température) et la puissance reçue du filament par rayonnement direct. Tenu compte de la présence du réflecteur qui, en raison de ses deux faces, renvoie vers l'ioniseur approximativement la moitié du rayonnement qu'il reçoit, la contribution du filament est au plus : $\frac{3}{4} V_{\mathrm{f}} I_{\mathrm{f}}$. Dans ces conditions, on observe la loi de Wien : $P \propto T^{4}$ lorsque la température de l'ioniseur est assez élevée pour que les pertes par rayounement soient prépondérantes devant les pertes par corduction thermique dans les colonnettes.

L'étude à une dimension (c'est-à-dire en négligeant le rayonnement latéral et les pertes par conduction) de l'équilibre thermique entre le réflecteur, le filament et l'ioniseur, conduit à la relation plus exacte :

$$
P_{\mathrm{e}}+\frac{4-\eta}{2(3-\eta)} P_{\mathrm{f}}=\frac{4-\eta}{3-\eta} \eta \sigma S T^{4}
$$

où l'on a considéré comme égales les émissivités $\eta$ du tungstène et du tantale; $\sigma$ est la constante de Stefan et $S$ la surface utile de l'ioniseur $\left(14 \times 20=280 \mathrm{~mm}^{2}\right)$. La partie rectiligne de la courbe de la figure 4 au-dessus de $2300 \mathrm{~K}$ correspond en fait à l'équation :

$$
P_{\mathrm{e}}+0,7 P_{\mathrm{f}}=6,5 \times 10^{-12} T^{4} .
$$

De sa pente, on déduit $\eta \simeq 0,3$, valeur normale à ces températures pour une surface de tungstène non particulièrement polie.

Des pointés effectués en différents endroits au voisinage du sommet de l'ioniseur font fréquemment apparaître un gradient de température qui peut atteindre $20 \mathrm{~K} / \mathrm{mm}$ sur plusieurs millimètres. C'est là une conséquence fâcheuse, mais inéluctable, du chauffage en régime de charge d'espace : si l'on néglige la conduction thermique dans l'ioniseur, une variation $\Delta e$ de la distance filament-ioniseur (due à leurs déformations) produit une variation $\Delta T$ de la température de l'ioniseur, telle que :

$$
\frac{\Delta T}{T}=-\frac{1}{2} \frac{\Delta e}{e}
$$

et, à $3000 \mathrm{~K}$, il suffit que $e$ varie de $20 \mu \mathrm{m}$ pour que $\Delta T$ atteigne $30 \mathrm{~K}$. La situation est cependant moins catastrophique qu'il n'y paraît, car la conduction thermique atténue notablement cet effet. En tout état de cause, on cherche à annuler le gradient de température au voisinage du sommet de l'ioniseur en disposant des cales sur les extrémités des colonnettes de montage, de façon à accroître légèrement l'écart filament-ioniseur du côté trouvé le plus chaud. Mais il est bien évident que l'accumulation des corrections et des imprécisions d'étalonnage ne permet pas de garantir la précision de la mesure absolue de la température du sommet de l'ioniseur à mieux de quelques pour cent.

4.3 SPECTRE DE MASSE RÉSIDUEL. - Un exemple de spectre de masse résiduel, c'est-à-dire relevé en l'absence de bombardement, est présenté à la figure 5 pour la température d'ioniseur $2700 \mathrm{~K}$. Les ions parasites formés ont trois origines possibles :

- l'ioniseur lui-même (tungstène et impuretés alcalines). Bien que présents à l'état de traces seulement, les métaux alcalins ( $\mathrm{Li}, \mathrm{Na}$ et surtout $\mathrm{K}$ ) constituent l'essentiel de l'émission propre de l'ioniseur en raison de leur énergie d'ionisation très basse. Le centrage de l'ensemble extraction-spectromètre par rapport à l'ioniseur est effectué en maximalisant le courant de l'une de ces espèces ioniques;

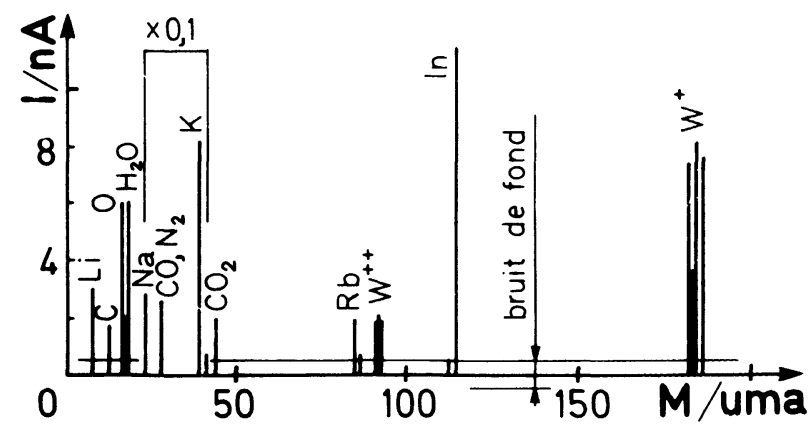

Fig. 5. - Spectre de masse résiduel à $2700 \mathrm{~K}$.

[Residual mass spectrum at $2700 \mathrm{~K}$.] 
- les parois de l'appareil qui, après un fonctionnement prolongé, sont contaminées par les matériaux des échantillons antérieurement bombardés (indium dans le cas présent);

- le gaz résiduel (eau, air, composés carbonés). Une partie de ces ions, en particulier les ions moléculaires, sont formés, non sur l'ioniseur, mais par bombardement électronique du gaz résiduel. Certains des électrons émis par le filament de chauffage, au lieu d'atteindre l'ioniseur, passent par les côtés et provoquent l'ionisation du gaz résiduel en dehors de l'axe du système.

Les ions $\mathrm{W}^{+}$n'apparaissent pratiquement qu'audessus de $2400 \mathrm{~K}$. La mesure de leur intensité d'émission est particulièrement intéressante, car très sensible à la température. On dispose ainsi d'un thermomètre interne qui permet de reproduire avec précision la température au voisinage du sommet de l'ioniseur, d'une expérience à une autre.

Tenu compte de la probabilité d'ionisation d'un atome de tungstène évaporé, sensiblement donnée par la formule (1) avec $V=7,98 \mathrm{eV}$, et de l'expression de la pression de vapeur d'un solide :

$$
p=\frac{Q_{0}}{Q_{\mathrm{s}}}\left(\frac{2 \pi m}{h^{2}}\right)^{3 / 2} \frac{k^{5 / 2} \theta^{3}}{e T^{1 / 2}} \exp \left(-\frac{\varepsilon_{\mathrm{d}}}{k T}\right)
$$

où $Q_{\mathrm{s}}$ tient lieu de fonction de partition électronique d'un atome dans le métal, $\theta$ est la température de Debye du tungstène et $\varepsilon_{\mathrm{d}}$ son énergie de sublimation $(8,7 \mathrm{eV})$, on établit aisément que le flux d'ions $\mathrm{W}^{+}$émis vaut :

$$
n\left(\mathrm{~W}^{+}\right)=\frac{p}{(2 \pi m k T)^{1 / 2}} \frac{Q_{\mathrm{i}}}{Q_{0}} \exp \left(-\frac{V-W}{k T}\right)
$$

soit :

$$
n\left(\mathrm{~W}^{+}\right)=\frac{Q_{\mathrm{i}}}{Q_{\mathrm{s}}} \frac{2 \pi m k^{2} \theta^{3}}{e h^{3}} \frac{1}{T} \exp \left(-\frac{\varepsilon_{\mathrm{i}}}{k T}\right)
$$

en introduisant l'énergie de sublimation à l'état ionisé $\varepsilon_{\mathrm{i}}$, donnée par la relation de Schottky [2] :

$$
\varepsilon_{\mathrm{i}}=\varepsilon_{\mathrm{d}}+V-W \simeq 12,1 \mathrm{eV} .
$$

Le courant d'ions $\mathrm{W}^{+}$mesuré à la sortie vaut finalement :

$$
I\left(\mathrm{~W}^{+}\right)=q s G n\left(\mathrm{~W}^{+}\right)
$$

où, comme au paragraphe $3.2, s$ est l'aire théorique intégralement imagée et $G$ le gain du multiplicateur pour les ions $\mathrm{W}^{+}$.

Comme $s$ varie en $1 / T$, la relation entre $\log T^{2} I\left(\mathrm{~W}^{+}\right)$et $1 / T$ doit etre une droite de pente $-\varepsilon_{\mathrm{i}} / k$, en négligeant la variation avec $T$ du rapport $Q_{\mathrm{i}} / Q_{\mathrm{s}}$. C'est effectivement ce que montre la figure 6 où la mesure de $I\left(\mathrm{~W}^{+}\right)$porte sur l'isotope 186 (d'abondance $28,5 \%$, bien séparé des autres), et d'où l'on déduit : $\varepsilon_{\mathrm{i}}=12,3 \pm 0,4 \mathrm{eV}$, en accord avec la

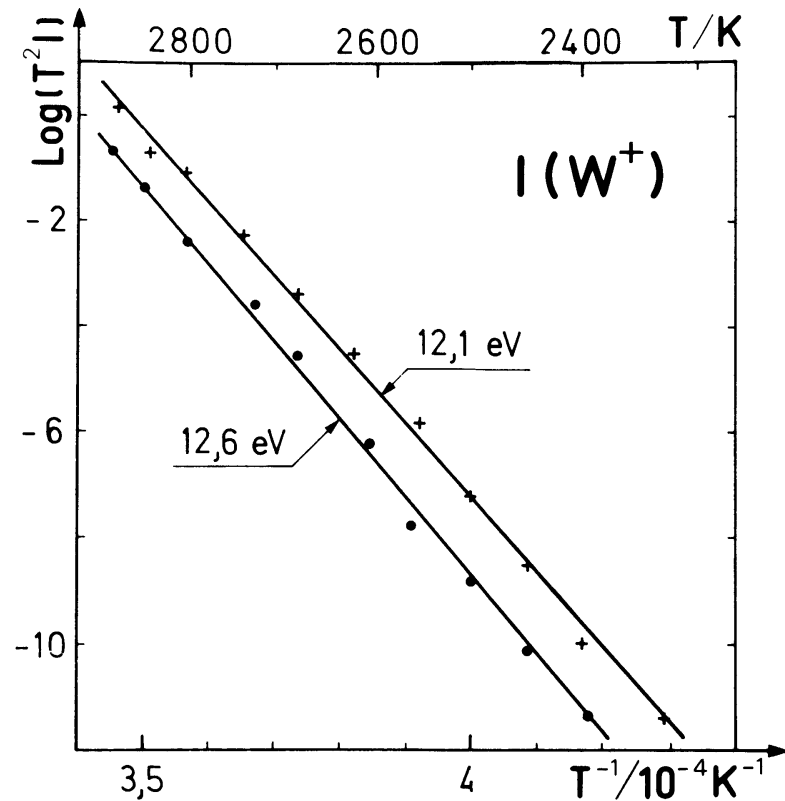

Fig. 6. - Variation avec la température de l'émission des ions $\mathrm{W}^{+}$ évaporés thermiquement (séries de mesures sur deux ioniseurs différents).

[Variation of the thermally evaporated $\mathrm{W}^{+}$ion emission versus the temperature (measurements on two different ionizing surfaces).]

prévision théorique ci-dessus et avec les résultats obtenus par Zandberg et al. [17] : $11,8 \pm 0,4 \mathrm{eV}$, et par Scheer et Fine [18] : 12,1 $\pm 0,2 \mathrm{eV}$.

Les mêmes auteurs [17] ont estimé le flux d'ions ${ }^{186} \mathrm{~W}^{+}$désorbés à $2700 \mathrm{~K}$ à la valeur :

$$
q n\left(\mathrm{~W}^{+}\right) \simeq 5 \mathrm{pA} / \mathrm{mm}^{2} .
$$

Or, à cette température, le courant mesuré d'ions $\mathrm{W}^{+}$ accélérés à $20 \mathrm{eV}\left(n=1,3\right.$ cycle H.F. $\left./ r_{0}\right)$ et analysés à la résolution unitaire $(\Delta M=1): R=186$, atteint 2 à $12 \mathrm{nA}$, tandis que l'aire théorique intégralement imagée peut être estimée à $0,4 \mathrm{~mm}^{2}$ (voir calculs en annexe 2). Par ailleurs, le gain $G$ du multiplicateur pour les ions $\mathrm{W}^{+}$, mesuré selon la technique de modulation exposée au paragraphe 2 , est de l'ordre de $2 \times 10^{4}$, valeur faible, mais plausible pour un multiplicateur à 11 dynodes souvent remis à l'air en quatre ans de service. On en déduit, à l'aide de la formule (5), l'estimation $q n\left(\mathrm{~W}^{+}\right) \simeq 1 \mathrm{pA} / \mathrm{mm}^{2}$, en accord raisonnable avec l'évaluation précédente, tenu compte des approximations dans le calcul de $s$ et de la grande sensibilité de $I\left(\mathrm{~W}^{+}\right)$à la valeur exacte de la température de l'ioniseur. De la formule (5), on déduit en effet :

$$
\frac{\Delta I\left(\mathrm{~W}^{+}\right)}{I\left(\mathrm{~W}^{+}\right)}=\left(\frac{\varepsilon_{\mathrm{i}}}{k T}-1\right) \frac{\Delta T}{T} .
$$

A $2700 \mathrm{~K}$, une variation de température de $5 \mathrm{~K}$ suffit pour modifier de $10 \%$ l'intensité $I\left(\mathrm{~W}^{+}\right)$. La mesure du courant d'ions $\mathrm{W}^{+}$permet donc un repérage fidèle et très sensible de la température de l'ioniseur, 
dans la mesure où la collection des ions émis n'est pas perturbée par d'imprévisibles déformations du ruban ioniseur.

5. Ionisation thermique de l'indium. -5.1 INFLUENCE DE LA TEMPÉRATURE DE L'IONISEUR. - Un échantillon d'indium est bombardé par des ions $\mathrm{A}^{+}$ de $10 \mathrm{keV}$ et le courant $I\left(\mathrm{In}^{+}\right)$est mesuré pour différentes valeurs de la température de l'ioniseur. Le coefficient d'ionisation $\beta$ est proportionnel à la quantité $T I\left(\operatorname{In}^{+}\right) / I_{1}$ (facteur $T$ pour tenir compte de la variation de $s$ en $1 / T$; cf. $\S 3.2$ ), dont le logarithme est porté à la figure 7 en fonction de $1 / T$ pour différentes valeurs du courant primaire dans le vide limite de l'appareil, et pour $I_{1}=10 \mu \mathrm{A}$ à une pression d'oxygène de $2 \times 10^{-6}$ torr. Les mesures ont été effectuées sur l'isotope 115 d'abondance $95,8 \%$. Selon toute vraisemblance, l'émission ne comporte pratiquement que les ions simples $\mathrm{In}^{+}$, comme c'est le cas pour l'aluminium [6,9]. Ce point n'a cependant pu être vérifié, car le domaine de masses de notre spectromètre est limité à $M=200$.

Les courbes de la figure 7 présentent la même allure générale que les courbes obtenues avec un jet thermique d'atomes d'indium [8]. Elles comportent en particulier une partie cornmune rectiligne à haute

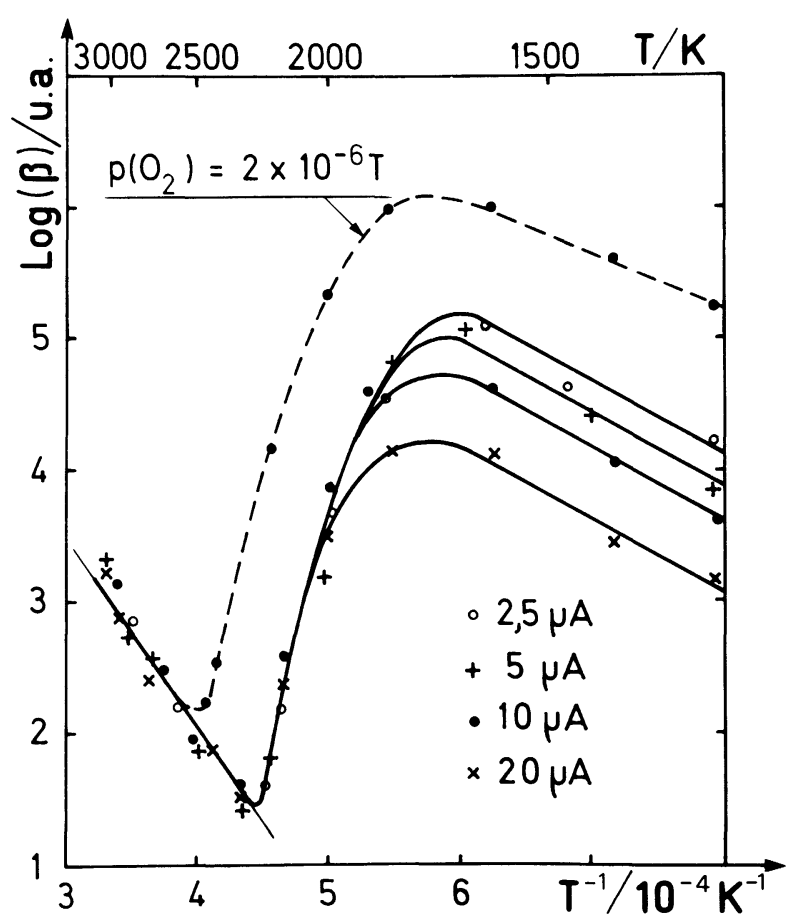

Fig. 7. - Variation, avec la température, du coefficient d'ionisation $\beta$ de l'indium, mesuré par $T I\left(\operatorname{In}^{+}\right) / I_{1}$, pour différentes valeurs du courant primaire $I_{1}$ (en trait continu : $p\left(\mathrm{O}_{2}\right)<2 \times 10^{-7}$ torr ; en trait tireté : $p\left(\mathrm{O}_{2}\right)=2 \times 10^{-6}$ torr).

[Variation, versus the temperature, of the ionization coefficient $\beta$ measured by $T I\left(\mathrm{In}^{+}\right) / I_{1}$, for different values of the primary current $I_{1}$ (solid lines : $p\left(\mathrm{O}_{2}\right)<2 \times 10^{-7}$ torr ; interrupted line :

$$
\left.\left.p\left(\mathrm{O}_{2}\right)=2 \times 10^{-6} \text { torr }\right) .\right]
$$

température dont la pente, à la précision des mesures, est bien celle prévue par la loi de Saha-Langmuir avec $\dot{V}=5,78 \mathrm{eV}$ et $W=4,6 \mathrm{eV}$ (la variation avec $T$ de la fonction de partition électronique $Q_{0}$ est négligeable sur le faible domaine de températures où la formule (1) peut être vérifiée). On remarque au passage que, bien que la cible d'indium soit proche de sa température de fusion, le rendement de pulvérisation n'en semble guère affecté (voir $\S 5.2$ ).

Au-dessous de $2300 \mathrm{~K}$, l'émission s'accroît très appréciablement. C'est là un comportement bien connu en ionisation de surface, qui a pour origine l'oxydation de l'ioniseur par l'oxygène ou l'eau du gaz résiduel. De fait, la courbe tracée à $2 \times 10^{-6}$ torr de pression d'oxygène s'écarte de la droite de SahaLangmuir dès $2500 \mathrm{~K}$. Le travail de sortie du tungstène recouvert d'oxygène peut monter jusqu'à $6,8 \mathrm{eV}$ [16], valeur à laquelle le coefficient d'ionisation $\beta$ devrait être proche de sa valeur maximale.

Ce raisonnement suppose toutefois que la surface de l'ioniseur n'est pas appréciablement contaminée par l'indium en régime permanent, c'est-à-dire que le flux incident $N$ d'atomes d'indium n'est pas trop important, de sorte que la condition $N \tau \ll 10^{13} \mathrm{at} / \mathrm{mm}^{2}$ (voir $§ 3.1$ ) reste satisfaite à la température considérée. Il semble bien que ce ne soit plus le cas au-dessous de $1800 \mathrm{~K}$, ce qui se traduit à la figure 7 par une remontée de $\beta$ d'autant plus limitée que le courant primaire $I_{1}$ (et donc $N$ ) est plus grand. Aux températures encore plus basses, l'ioniseur est de plus en plus contaminé par l'indium et son efficacité diminue progressivement. La reproductibilité des mesures devient mauvaise : non seulement, il faudrait pouvoir contrôler plus soigneusement la nature et la pression $\mathrm{du}$ gaz résiduel, ainsi que l'intensité du courant primaire, mais aussi un phénomène d'hystérésis bien connu [2] se manifeste : quand on opère à températures décroissantes, la chute de l'émission se produit à plus basse température que lorsqu'on opère à températures croissantes.

L'étude de la variation du courant d'ions In $^{+}$ en fonction du courant d'ions $\mathrm{W}^{+}$évaporés est possible dans l'intervalle $2300-3000 \mathrm{~K}$, domaine de températures où l'oxydation de l'ioniseur est exclue aux pressions utilisées. En ne conservant que les termes exponentiels dans les expressions (2) et (5), les autres termes pouvant être considérés comme à peu près constants sur un faible intervalle de températures, on établit facilement la relation :

$$
\log \frac{I\left(\operatorname{In}^{+}\right)}{I_{1}}=\frac{V-W}{\varepsilon_{\mathrm{i}}} \log I\left(\mathrm{~W}^{+}\right)+\text {Cte } .
$$

De fait, le tracé expérimental de cette relation, présenté à la figure 8 , conduit à une droite dont la pente : 0,12 $\pm 0,02$ est en bon accord avec la valeur théorique : 0,10 . La valeur trouvée pour la constante dépend cependant quelque peu de l'ioniseur, comme c'était déjà le cas pour $I\left(\mathrm{~W}^{+}\right)$(cf. $\S 4.3$ et Fig. 6). 


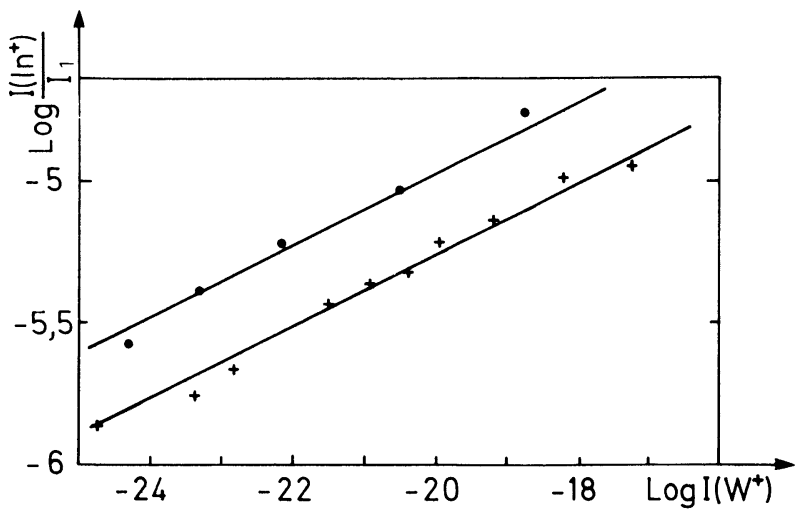

Fig. 8. - Variation de $\log I\left(\operatorname{In}^{+}\right) / I_{1}$ en fonction de $\log I\left(\mathrm{~W}^{+}\right)$ (séries de mesures sur deux ioniseurs différents).

[Variation of $\log I\left(\mathrm{In}^{+}\right) / I_{1}$ versus $\log I\left(\mathrm{~W}^{+}\right)$(measurements on two different ionizing surfaces).]

5.2 Evaluation du Courant D'IONS $I\left(\mathrm{In}^{+}\right)$. Nous vous proposons d'évaluer, à l'aide de la formule (2), l'intensité $I\left(\operatorname{In}^{+}\right)$que l'on devrait observer pour une température d'ioniseur égale à $2700 \mathrm{~K}$. A cette température, l'oxydation du tungstène par le gaz résiduel est tout à fait négligeable et $W=4,60 \mathrm{eV}$ [8]. Tenu compte du premier niveau électronique excité de l'indium neutre (à $\left.2112,56 \mathrm{~cm}^{-1}\right)$, le rapport $Q_{\mathrm{i}} / Q_{0}$ des fonctions de partition électroniques vaut : 0,31 et le coefficient d'ionisation atteint $: \beta \simeq 2 \times 10^{-3}$.

L'aire théorique intégralement imagée est évaluée en annexe 2 à l'aide de la formule (3) pour des ions $\mathrm{In}^{+}$ émis à $2700 \mathrm{~K}$ et ayant une énergie de $20 \mathrm{eV}$ à leur entrée dans le quadrupôle. On obtient $: s \simeq 0,6 \mathrm{~mm}^{2}$ pour la résolution unitaire $R=115$.

A priori, le gain du multiplicateur fait intervenir le rendement électronique $\gamma$ de la première dynode et dépend donc de la nature des ions incidents. En principe, le rendement relatif dépend peu de l'énergie des ions $[19,20]$. Dans ces conditions, par comparaison de la valeur de $\gamma$ pour l'indium normalisée à l'argon à $5 \mathrm{keV}$ [20] et celle pour le tungstène normalisée au potassium à $8 \mathrm{keV}$ [21], et en utilisant les éléments $(\mathrm{Ca}, \mathrm{Sn}$ et $\mathrm{Pb})$ communs aux deux références, on trouve : $G\left(\operatorname{In}^{+}\right) / G\left(\mathrm{~W}^{+}\right) \simeq 1,3$. Par ailleurs, la courbe universelle proposée par Pottie et al. [20] conduit à la valeur : 1,2. En fait, nous avons obtenu pour les ions $\mathrm{In}^{+}$un gain à peine supérieur à celui trouvé pour les ions $\mathrm{W}^{+}$, sans doute parce que l'énergie des ions à leur arrivée sur la première dynode $(\simeq 3 \mathrm{keV})$ devient proche de l'énergie-seuil de l'émission cinétique [1]. Pour l'évaluation de $I\left(\mathrm{In}^{+}\right)$, nous adoptons la valeur mesurée : $G \simeq 2 \times 10^{4}$.

Le rendement de pulvérisation de l'indium, bombardé par des ions $\mathrm{A}^{+}$de $10 \mathrm{keV}$ sous une incidence de $45^{\circ}$, peut se calculer par la théorie de Sigmund [22], en tenant compte de la correction à apporter au paramètre $\alpha\left(M_{2} / M_{1}\right)$ de cette théorie pour $M_{2}>M_{1}$, d'après les résultats expérimentaux de Andersen et Bay [23]. On trouve ainsi $S \simeq 16$ atomes/ion, valeur un peu supérieure à celle calculée pour le cuivre dans les mêmes conditions de bombardement : 11 atomes/ion, alors que les valeurs expérimentales publiées pour ces deux métaux sont à peu près égales : respectivement 6,5 et 6 pour des ions $\mathrm{A}^{+}$ de $45 \mathrm{keV}$ à incidence normale [1] et 12,2 et 11,8 pour des ions $\mathrm{Kr}^{+}$de $45 \mathrm{keV}$ à incidence normale [24].

La mesure directe du rendement de pulvérisation est en principe possible avec notre appareillage, en déterminant, au microscope par exemple, la profondeur de la cuvette creusée par le bombardement ionique en un temps donné. En fait, la mesure absolue de $S$ suppose la connaissance du volume de matière pulvérisée ; elle est peu précise en raison de la nonplanéité du fond de la cuvette, tant à cause de la non-uniformité du bombardement que des variations de la vitesse d'érosion selon les orientations des divers microcristaux. Tout au plus peut-on vérifier que l'ordre de grandeur est correct. Les mesures relatives sont plus significatives, si l'on prend la précaution d'ajuster les temps de bombardement de manière à obtenir des profondeurs décapées à peu près égales. Nous trouvons ainsi des valeurs de $S$ très voisines pour le cuivre et l'indium, en accord avec les expériences citées plus haut, et adoptons finalement la valeur approchée $: S \simeq 10$ atomes/ion.

Par la même occasion, nous avons vérifié que le rendement de pulvérisation de l'indium n'est guère affecté (moins de $20 \%$ ) par la température de la cible. En fait, la mesure de $S$ n'est significative que jusqu'à une température d'ioniseur de l'ordre de $2800 \mathrm{~K}$. Au-delà, le rayonnement incident dépasse $10 \mathrm{~W} / \mathrm{cm}^{2}$ et conduit à un échauffement de la surface tel que le profil de la cuvette en est complètement modifié, vraisemblablement par capillarité. La qualité du contact thermique de l'échantillon avec le support refroidi joue évidemment un rôle essentiel dans cette limitation.

En rassemblant tous ces résultats dans la formule (2), on prévoit ainsi l'intensité $I\left(\mathrm{In}^{+}\right)=1,2 \times 10^{-6} \mathrm{~A}$, à $2700 \mathrm{~K}$ et pour un courant primaire d'intensité $I_{1} \simeq 10 \mu \mathrm{A}$. Les valeurs expérimentalement mesurées de $I\left(\operatorname{In}^{+}\right)$vont de $6 \times 10^{-8}$ à $2,3 \times 10^{-7} \mathrm{~A}$ et semblent donc de 5 à 20 fois trop faibles. Le désaccord entre valeur théorique et résultats expérimentaux est cependant du même ordre que celui trouvé lors de l'évaluation de $I\left(\mathrm{~W}^{+}\right)$(cf. § 4.3). Ainsi les valeurs expérimentalement obtenues pour le rapport $I\left(\mathrm{In}^{+}\right) / I\left(\mathrm{~W}^{+}\right)$se répartissent-elles autour de la valeur théorique : 30 , avec une dispersion importante provenant pour une large part de la méconnaissance de la valeur exacte de la température de l'ioniseur. Il est donc vraisemblable que la transmission globale du spectromètre est surestimée de près d'un facteur 10 par les calculs approchés des annexes 1 et 2 , tant $\mathrm{du}$ fait des approximations de calcul que du fait d'éventuels défauts d'alignement. 
6. Conclusion. - L'appareillage que nous venons de décrire pourrait certes être amélioré. Nos efforts se sont tout d'abord portés sur la réalisation d'un montage filament-ioniseur permettant d'atteindre les hautes températures dans des conditions raisonnables de reproductibilité et de stabilité. Ce résultat a été atteint, mais des progrès seraient encore souhaitables en ce qui concerne la fiabilité de l'ioniseur, dont les déformations à chaud restent imprévisibles et sont trop souvent bien fâcheuses. Il serait sans doute intéressant d'alimenter le filament à partir d'un point milieu vers ses deux extrémités, les deux parties travaillant en parallèle, pour établir un régime de chauffage symétrique et mieux uniformiser la température au voisinage du sommet de l'ioniseur.

A plus long terme, il faudrait chercher à accroître la sensibilité du montage pour pouvoir mettre en œuvre une sonde ionique de faible diamètre, afin de mieux localiser la région analysée. L'ioniseur devrait naturellement être réalisé en un matériau de haute pureté pour que son spectre résiduel ne vienne pas trop perturber l'analyse [6] ; le fonctionnement en ultra-vide pourrait aussi s'avérer nécessaire. Il conviendrait de diminuer autant que possible la distance cible-ioniseur, en réduisant les dimensions du système d'extraction (d'autres géométries pourraient vraisemblablement être envisagées). Mais surtout, il faudrait utiliser un spectromètre quadrupolaire de plus grande taille, équipé d'un multiplicateur d'électrons de gain élevé, disposé latéralement ou en dehors de l'axe du quadrupôle. On trouvera dans un autre article une étude détaillée des possibilités et des limitations de cette méthode d'analyse [25].

Quoi qu'il en soit, cet appareillage, tel qu'il a été décrit, a d'ores et déjà permis de répondre à la principale question posée par la méthode : une seule interaction avec la surface ionisante semble suffire pour réaliser l'équilibre thermodynamique, pour les états électroniques tout au moins. Cette conclusion découle de la similitude des résultats obtenus dans l'ionisation de l'indium, que ce soit après évaporation thermique [8] ou après pulvérisation ionique. Seule l'analyse en énergie des ions formés sur l'ioniseur et l'étude de la répartition angulaire de leur émission permettraient de savoir ce qu'il en est de la thermalisation des énergies cinétiques.

Remerciements. - Les auteurs remercient vivement G. Blaise pour de fructueuses conversations et la communication de résultats non publiés. Ils tiennent à remercier tout particulièrement leur technicien, J.-P. Leroux, qui assure avec compétence et dévouement le montage et la maintenance de l'appareillage. Ils ont apprécié aussi l'aide efficace de P. Viaris de Lesegno pour les améliorations électroniques et les calculs sur machine.

Annexe 1. - PropriÉtés OPTIQUES DU SYSTÈme D'EXTRACTION ET DE TRANSFERT. - Si l'on admet que l'équilibre thermique est réalisé entre l'ioniseur et les particules désorbées, les ions formés ont leurs énergies cinétiques initiales $\varepsilon$ distribuées selon une loi en $\varepsilon \exp (-\varepsilon / k T)$, tandis que leur répartition angulaire suit la loi en cosinus. L'énergie la plus probable des ions émis est de l'ordre de $k T$, soit 0,15 à $0,26 \mathrm{eV}$ de 1700 à $3000 \mathrm{~K}$; elle est petite devant l'énergie $E=q\left(V_{\mathrm{A}}-V_{\mathrm{B}}\right)$ acquise dans le champ électrique accélérateur à symétrie approximativement sphérique qui règne entre l'ensemble ioniseurwehnelt (assimilé à une sphère de rayon $a=20 \mathrm{~mm}$ au potentiel $V_{\mathrm{A}}$ ) et l'électrode d'extraction (de rayon $b=7 \mathrm{~mm}$ au potentiel $V_{\mathbf{B}}$ ).

Si les ions étaient formés sur l'ioniseur avec une énergie initiale quasi nulle, ils convergeraient tous vers le centre $O$ des sphères (Fig. 9), l'effet de charge d'espace étant bien entendu supposé négligeable. Toutefois, le passage par un trou circulaire d'une région de champ accélérateur à une région de champ nul implique nécessairement une région de transition avec un champ électrique radial [26]. L'entrée B du canal d'extraction est ainsi équivalente à une lentille divergente d'axe AO et de distance focale : $4 b(a-b) / a$, que l'on peut considérer comme mince dans la mesure où l'on admet que le rayon $d$ du trou d'extraction $(2 \mathrm{~mm})$ est petit devant $b$.

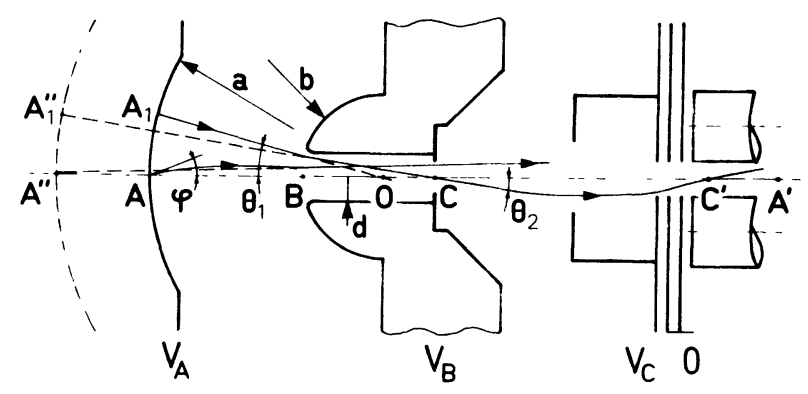

Fig. 9. - Schéma optique du montage (tracé pour $T=2700 \mathrm{~K}$; $\left.V_{\mathrm{A}}=20 \mathrm{~V}, V_{\mathrm{B}}=0, V_{\mathrm{C}}=-120 \mathrm{~V}\right)$.

[Optical scheme of the apparatus (drawn for $T=2700 \mathrm{~K}$; $\left.V_{\mathrm{A}}=20 \mathrm{~V}, V_{\mathrm{B}}=0, V_{\mathrm{C}}=-120 \mathrm{~V}\right)$.]

Il en résulte que le faisceau des ions émis avec une énergie initiale quasi nulle d'une aire voisine $\mathrm{du}$ sommet $\mathrm{A}$ de l'ioniseur présente une région d'amincissement en un cross-over $\mathrm{C}$ situé un peu au-delà de $\mathrm{O}$, tel que :

$$
\overline{\mathrm{OC}}=\frac{a b}{3 a-4 b} \simeq 4,4 \mathrm{~mm} .
$$

Une trajectoire issue du point $A_{1}$ en dehors de l'axe, faisant initialement l'angle $\theta_{1}$ petit avec l'axe, se prolongerait en une trajectoire rectiligne inclinee de l'angle $\theta_{2}$ tel que :

$$
\frac{\theta_{2}}{\theta_{1}}=\frac{3 a-4 b}{4(a-b)} \simeq 0,61
$$


à condition toutefois que $\theta_{1}$ soit inférieur à Arc $\sin d / b \simeq 17^{\circ}$, ce qui limite l'aire théorique de collection à un disque de centre $\mathrm{A}$ et de diamètre voisin de $12 \mathrm{~mm}$.

En fait, un ion émis du sommet $\mathrm{A}$ de l'ioniseur avec l'énergie initiale $\varepsilon$, dans une direction faisant l'angle $\varphi$ avec l'axe $\overline{\mathrm{AO}}$, décrit une trajectoire elliptique dans le champ accélérateur à symétrie sphérique en $1 / r^{2}$. Avec ces conditions initiales, l'équation de l'ellipse en coordonnées polaires $(r, \theta)$ de centre $\mathrm{O}$, s'écrit :

$$
r(\theta)=2 \frac{\varepsilon}{E} \frac{a}{b}(a-b) \frac{\sin ^{2} \varphi}{1+e \cos \left(\theta-\theta_{0}\right)}
$$

avec :

$$
\left\{\begin{aligned}
e & =\left[1-2 \frac{\varepsilon}{E} \frac{a-b}{b} \sin ^{2} \varphi\left(2-2 \frac{\varepsilon}{E} \frac{a-b}{b}\right)\right]^{1 / 2} \\
\operatorname{tg} \theta_{0}= & \frac{2 \frac{\varepsilon}{E} \frac{a-b}{b} \sin \varphi \cos \varphi}{1-2 \frac{\varepsilon}{E} \frac{a-b}{b} \sin ^{2} \varphi} .
\end{aligned}\right.
$$

Un calcul approché est possible si l'on tient compte de ce que $\varepsilon / E$ est petit ; les résultats obtenus ont été vérifiés et précisés par calcul exact à l'ordinateur. Limitant les développements en série au terme en $\sqrt{\varepsilon / E}$, on trouve que l'ion arrive à l'électrode d'extraction à la distance $\rho$ de l'axe telle que :

$$
\rho \simeq 2(a-b) \sqrt{\frac{\varepsilon}{E}} \sin \varphi\left(1-\sqrt{\frac{\varepsilon}{E}} \cos \varphi\right) .
$$

La direction de sa vitesse fait alors avec l'axe l'angle $\psi$ petit tel que :

$\operatorname{tg} \psi \simeq-\frac{a-2 b}{b} \sqrt{\frac{\varepsilon}{E}} \sin \varphi\left(1-2 \frac{a-b}{a-2 b} \sqrt{\frac{\varepsilon}{E}} \cos \varphi\right)$

et le prolongement rectiligne de la trajectoire de l'ion irait recouper l'axe au point $\mathrm{A}^{\prime}$, situé bien au-delà de $\mathrm{O}$, tel que :

$$
\overline{\mathrm{BA}^{\prime}}=-\frac{\rho}{\operatorname{tg} \psi} \simeq 2 b \frac{a-b}{a-2 b}\left(1+\frac{a}{a-2 b} \sqrt{\frac{\varepsilon}{E}} \cos \varphi\right) .
$$

Mais l'effet de lentille divergente à l'entrée dans l'électrode d'extraction déplace le point de convergence au point $A^{\prime \prime}$ tel que :

$$
\overline{\mathrm{BA}^{\prime \prime}} \simeq 4 b \frac{a-b}{a-4 b}\left(1+\frac{2 a}{a-4 b} \sqrt{\frac{\varepsilon}{E}} \cos \varphi\right)
$$

et l'angle $\psi^{\prime}$ avec l'axe de la trajectoire réellement suivie au-delà de l'électrode d'extraction est :

$$
\begin{aligned}
\psi^{\prime} & =-\frac{\rho}{\overline{\mathrm{BA}^{\prime \prime}}} \\
& \simeq-\frac{a-4 b}{2 b} \sqrt{\frac{\varepsilon}{E}} \sin \varphi\left(1-\frac{3 a-4 b}{a-4 b} \sqrt{\frac{\varepsilon}{E}} \cos \varphi\right)
\end{aligned}
$$

(le calcul de $\overline{\mathrm{BA}^{\prime \prime}}$ s'effectue à partir de $\overline{\mathrm{BA}^{\prime}}$ en conservant la distance focale : $4 b(a-b) / a$ calculée pour $\varepsilon=0$; la correction introduite par $\varepsilon \neq 0$ est en effet en $\varepsilon / E$, alors que nous avons convenu de limiter les calculs au terme en $\sqrt{\varepsilon / E}$.

Après accélération, un faisceau d'ions issus du sommet A de l'ioniseur se transforme en un faisceau faiblement divergent d'ouverture $\psi^{\prime}$, qui semble provenir d'un point $\mathrm{A}^{\prime \prime}$ virtuel situé à gauche de $\mathrm{A}$, tel que :

$\overline{\mathrm{CA}^{\prime \prime}}=8 a b \frac{a-b}{(3 a-4 b)(a-4 b)}\left(1+\frac{3 a-4 b}{a-4 b} \sqrt{\frac{\varepsilon}{E}} \cos \varphi\right)$

et le rayon $c$ de ce faisceau, au niveau du cross-over $\mathbf{C}$, vaut

$$
c=-\psi^{\prime} \times{\overline{\mathrm{CA}^{\prime \prime}}}^{\prime \prime}=4 a \frac{a-b}{3 a-4 b} \sqrt{\frac{\varepsilon}{E}} \sin \varphi
$$

en accord avec la relation de Lagrange-Helmholtz :

$$
\sqrt{\varepsilon} \times a \theta_{1} \times \sin \varphi=\sqrt{E} \times c \times \theta_{2} .
$$

Avec une loi d'émission en $\cos \varphi$, il en résulte que le cross-over est uniformément éclairé, c'est-à-dire que la densité des ions d'énergie $\varepsilon$ est uniforme à l'intérieur du disque de rayon :

$$
4 a \frac{a-b}{3 a-4 b} \sqrt{\frac{\varepsilon}{E}} \text {. }
$$

Les ions issus d'un point $A_{1}$ de l'ioniseur, en dehors de l'axe, mais voisin du sommet $A$ et repéré par l'angle $\theta_{1}$, définissent un faisceau élémentaire qui, après accélération, admet pour direction moyenne la droite passant par $\mathrm{C}$ et faisant avec l'axe $\overline{\mathrm{AO}}$ l'angle $\theta_{2} \simeq 0,61 \theta_{1}$, et dont l'ouverture, très petite, est égale à $\psi^{\prime}$. A cause de la symétrie sphérique du champ accélérateur, les ions sembleront provenir d'un point $\mathrm{A}_{1}^{\prime \prime}$ situé dans la direction $\theta_{2}$ à la distance $\overline{\mathrm{CA}}_{1}^{\prime \prime}=\overline{\mathrm{CA}}^{\prime \prime}$. Toutefois, lorsque $\theta_{1}$ dépasse la valeur limite : $\operatorname{Arcsin}(d-\rho) / b$, une fraction des ions vient heurter l'électrode d'extraction et est éliminée $\mathrm{du}$ faisceau. Heureusement, cette élimination porte d'abord sur les ions émis aux grands angles, dont la contribution est peu importante. Pour $\rho \ll d$, la fraction éliminée atteint $50 \%$ quand le point d'émission $\mathrm{A}_{1}$ se situe au bord de l'aire théorique de collection, soit à $6 \mathrm{~mm}$ de $\mathrm{A}$. Quand $\rho$ et $d$ sont voisins, l'élimination à $50 \%$ se produit pour $\mathrm{AA}_{1} \simeq 5 \mathrm{~mm}$.

Le système d'extraction des ions recueille donc essentiellement les ions émis de la région centrale de l'ioniseur (disque de diamètre voisin de $10 \mathrm{~mm}$ ). Après accélération, ces ions constituent un faisceau dont l'ouverture est fixée à $9^{\circ}$ environ par le rayon de la plage d'émission collectée à $50 \%$ au moins, et qui présente au point $\mathrm{C}$ un cross-over dont le rayon $c$, au plus égal à $d$ (soit $2 \mathrm{~mm}$ ), dépend de l'énergie initiale $\varepsilon$ et de la direction $\varphi$ d'émission des ions, selon la formule (6). 
Il serait souhaitable que l'optique de transfert constituée par les électrodes de la source d'ions de l'analyseur quadrupolaire forme l'image $C^{\prime}$ du crossover $\mathrm{C}$ à l'intérieur du quadrupôle, à une distance de l'ordre de $0,7 r_{0}$, soit $2 \mathrm{~mm}$ de l'entrée dans le champ $[12,14]$. Un calcul sur machine des trajectoires ioniques a été effectué avec $V_{\mathrm{A}}=20 \mathrm{~V}$ (de manière à avoir $n=1$ cycle H.F. $/ r_{0}$ pour des ions $\left.\operatorname{In}^{+}\right), V_{\mathrm{B}}=0$ et $-30 \mathrm{~V}, V_{\mathrm{C}}=-120 \mathrm{~V}\left(V_{\mathrm{C}}\right.$ est le potentiel de la cage de la source d'ions, dont les autres électrodes sont reliées à la masse). Les résultats obtenus sont voisins pour les deux valeurs de $V_{\mathrm{B}}$ en ce qui concerne la position du point de focalisation (à $8 \mathrm{~mm}$ de l'entrée du champ) et le grandissement linéaire, de l'ordre de 0,6 ; les grandissements angulaires valent respectivement 1,7 et 2,8 .

Concernant l'acceptance du quadrupôle, on établit, à l'aide des tableaux II et III de la référence [14], que, pour $n=1$ cycle H.F. $/ r_{0}$, pour la résolution $R=M / \Delta M=55$ et tenu compte de la taille différente du quadrupôle utilisé, les angles limites d'injection pour la transmission $50 \%$ valent : $\theta_{50}^{x} \simeq 9^{\circ}$ et $\theta_{50}^{y} \simeq 6^{\circ}$, à condition que les rayons du crossover soient inférieurs à $x_{\mathrm{m}} \simeq 0,4 \mathrm{~mm}$ et $y_{\mathrm{m}} \simeq 0,6 \mathrm{~mm}$. Dans ces conditions, c'est le quadrupôle qui sélectionne les trajectoires ioniques transmises et il est de peu d'importance que le point de focalisation de l'optique de transfert ne se situe pas tout à fait à l'emplacement optimal. Le cross-over utile, ramené au niveau de $\mathrm{C}$, a une forme elliptique; son petit axe vaut approximativement : $x_{\mathrm{m}} / 0,6 \simeq 0,7 \mathrm{~mm}$ et son grand axe : $y_{\mathrm{m}} / 0,6 \simeq 1 \mathrm{~mm}$. On peut sans inconvénient placer un diaphragme de $2,4 \mathrm{~mm}$ de diamètre au niveau de $C$, dans le canal de l'électrode d'extraction, pour limiter la contamination du quadrupôle et réduire le flux de photons sur le multiplicateur.

Annexe 2. - Evaluation de l'aire imagée. L'aire théorique intégralement imagée $s$ s'obtient à l'aide de la formule (3) établie au paragraphe 3.2 :

$$
s=\pi \frac{q V_{\mathrm{A}}}{k T} x_{\mathrm{m}} y_{\mathrm{m}} \theta_{50}^{x} \theta_{50}^{y} .
$$

Ainsi, pour la température d'ioniseur $T=2700 \mathrm{~K}$, pour la résolution $R=55$ et $n=1$ cycle H.F. $/ r_{0}$, on trouve $s \simeq 1,1 \mathrm{~mm}^{2}$ avec des ions $\mathrm{In}^{+}$de $20 \mathrm{eV}$, et $s \simeq 1,7 \mathrm{~mm}^{2}$ avec des ions $\mathrm{W}^{+}$de $32 \mathrm{eV}$. En fait, les mesures sont généralement effectuées avec $V_{\mathrm{A}}=20 \mathrm{~V}$ et la résolution unitaire $(\Delta M=1)$, c'est-àdire $R=115$ pour les ions $\mathrm{In}^{+}$et $R=186$ pour les ions $\mathrm{W}^{+}$. Comme le montre la figure 10 , où le courant $I\left(\mathrm{In}^{+}\right)$mesuré est porté en fonction de la résolution (avec normalisation à $s=1,1 \mathrm{~mm}^{2}$ pour $R=55$ ), la transmission globale du spectromètre varie grossièrement en $1 / R$ aux faibles résolutions, très inférieures à la résolution limite du quadrupôle [12] ; elle diminue d'un facteur de l'ordre de 1,8 quand la résolution passe de la valeur 55 à la valeur

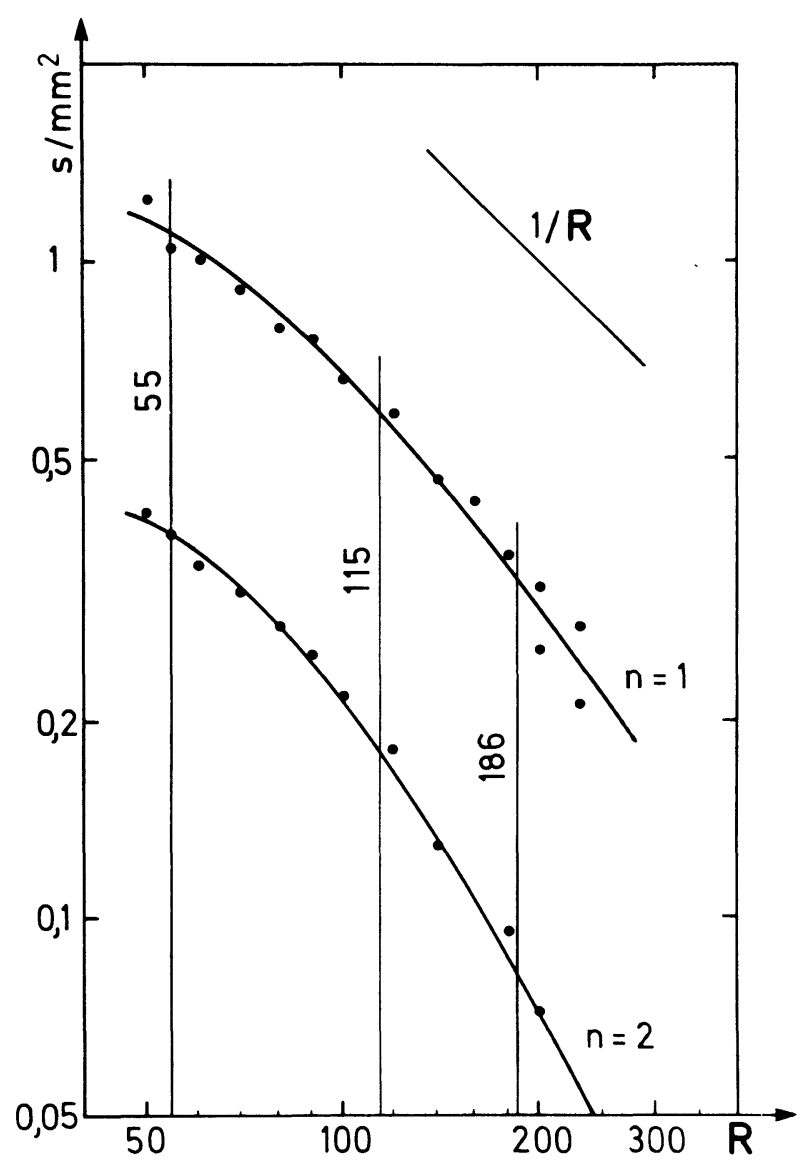

Fig. 10. - Variation, avec la résolution $R$, de la surface théorique intégralement imagée $s$, mesurée par le courant collecté et normalisée à $s=1,1 \mathrm{~mm}^{2}$ pour $R=55$ et $n=1$ cycle H.F. $/ r_{0}$.

[Variation of the fully imaged theoretical area $s$ versus the resolution $R$, measured by the collected ion current after normalization at $s: 1,1 \mathrm{~mm}^{2}$ for $R=55$ and $n=1$ R.F. cycle $/ r_{0}$.]

usuelle 115. C'est ainsi qu'est obtenue l'estimation $s\left(\operatorname{In}^{+}\right) \simeq 0,6 \mathrm{~mm}^{2}$ utilisée au paragraphe 5.2 .

La situation est plus compliquée pour les ions $\mathrm{W}^{+}$, car le chevauchement des isotopes 184 et 186 ne permet pas de prolonger la courbe transmissionrésolution au-dessous de $\Delta M \simeq 1,5(R \sim 120)$. Mais les ions $\mathrm{W}^{+}$de $20 \mathrm{eV}\left(n \simeq 1,3\right.$ cycles H.F. $\left./ r_{0}\right)$ sont à peine plus lents que les ions $\operatorname{In}^{+}$de même énergie, et l'acceptance du quadrupôle est maximale entre 1 et 2 cycles H.F. $/ r_{0}$ [12]. Dans ces conditions, on peut adopter pour les ions $\mathrm{W}^{+}$de $20 \mathrm{eV}$ la valeur approximative $s=1,2 \mathrm{~mm}^{2}$ à $R=55$. Comme les courbes transmission-résolution des ions $\mathrm{In}^{+}$et $\mathrm{W}^{+}$ (pour $R>120$ ) ont la même allure, et que, la transmission globale a chuté d'un facteur presque égal à 3 à la résolution 186, on aboutit à l'évaluation $s\left(\mathrm{~W}^{+}\right) \simeq 0,4 \mathrm{~mm}^{2}$ utilisée au paragraphe 4.3 .

Sur la même figure 10 , nous avons porté la courbe transmission-résolution relevée à $n=2$ cycles H.F. $/ r_{0}$, soit à $5 \mathrm{eV}$ pour $\mathrm{In}^{+}$et $8 \mathrm{eV}$ pour $\mathrm{W}^{+}$. $\mathrm{La}$ diminution observée de $s$ par rapport à $n=1$ cycle H.F. $/ r_{0}$ est de l'ordre de 3 à la résolution 
55 , alors que le calcul à partir des tableaux II et III de [14] conduit à un facteur voisin de 2 . Ces estimations de $s$ doivent être considérées comme des ordres de grandeur : l'hypothèse d'un champ accélérateur à symétrie sphérique est évidemment optimiste, et la détermination de la transmission, par un quadrupôle, d'un faisceau ionique relativement ouvert nécessite des calculs bien plus complexes [27].

Comme les angles limites d'ouverture $\theta_{2}$ au niveau de $C$ valent respectivement $5^{\circ}$ et $3,5^{\circ}$ (pour $V_{B}=0$ ) et $3^{\circ}$ et $2^{\circ}$ (pour $V_{\mathrm{B}}=-30 \mathrm{~V}$ ), l'aire $s^{\prime}$ effectivement imagée est bien plus grande que $s$. Sa valeur s'obtient en écrivant :

$$
s=s^{\prime} \sin \varphi_{x} \sin \varphi_{y}
$$

en donnant aux angles limites de collection $\varphi_{u}$ leurs valeurs maximales déduites de la formule (6) lorsque le rayon du cross-over atteint ses valeurs limites $u_{\mathrm{m}} / 0,6$. On trouve ainsi :

$$
s^{\prime}=\pi \frac{V_{\mathrm{A}}}{V_{\mathrm{A}}-V_{\mathrm{B}}}\left[\frac{2,4 a(a-b)}{3 a-4 b}\right]^{2} \theta_{50}^{x} \theta_{50}^{y} .
$$

L'aire effectivement imagée est un disque elliptique, allongé dans la direction $x$. Sa surface $s^{\prime}$ est indépendante de la température; pour des ions $\mathrm{In}^{+}$ de $20 \mathrm{eV}$ et $R=55$, elle vaut $20 \mathrm{~mm}^{2}$ (pour $V_{\mathrm{B}}=0$ ) et $8 \mathrm{~mm}^{2}$ (pour $V_{\mathrm{B}}=-30 \mathrm{~V}$ ). Les angles limites de collection $\varphi_{u}$ diminuent quand la température croît. A $2700 \mathrm{~K}$, ils valent $12^{\circ}$ et $17^{\circ}$ (pour $V_{\mathrm{B}}=0$ ) et $18^{\circ}$ et $27^{\circ}$ (pour $V_{\mathrm{B}}=-30 \mathrm{~V}$ ). Ces limites pour $s^{\prime}$ et $\varphi$ sont imposées par le quadrupôle et son optique de collection et de transfert; elles correspondent à une transmission à $50 \%$ des ions. Il est prudent de prévoir une certaine marge, ce qui explique la largeur relativement importante du ruban ioniseur.

Le fait de porter l'électrode d'extraction à $V_{\mathrm{B}}=-30 \mathrm{~V}$ au lieu de $V_{\mathrm{B}}=0$ ne change pas la valeur de $s$ et ne devrait donc avoir aucun effet sur le courant collecté $I$. On observe en fait un léger accroissement de $I$ auquel on peut trouver plusieurs raisons : ions plus rapides, donc moins sensibles aux champs parasites, trajectoires plus proches de l'axe ( $s^{\prime}$ plus petit), faisceau mieux focalisé au niveau du cross-over $\mathrm{C}$, thermalisation imparfaite des énergies cinétiques initiales $(\varepsilon>k T) \ldots$ Cet accroissement reste faible ; aussi, par commodité, les mesures sur $\mathrm{In}^{+}$et $\mathrm{W}^{+}$ont-elles été effectuées avec l'anode à la masse $\left(V_{\mathbf{B}}=0\right)$.

\section{Bibliographie}

[1] Kaminsky, M., Atomic and Ionic Impact Phenomena on Metal Surfaces (Springer Verlag, Berlin-Heidelberg-New York) 1965.

[2] Ionov, N. I., Progress in Surface Science édité par S. G. Davison (Pergamon Press, Oxford) 1 (1972) 237.

[3] Townsend, P. D., Kelly, J. C. et Hartley, N. E. W., Ion Implantation, Sputtering and their Applications (Academic Press, London) 1976.

[4] Bradley, R. C., Phys. Rev. 93 (1954) 719.

[5] Castaing, R., Blaise, G. et Quettier, R., VII Congrès International sur l'Optique des Rayons $X$ et la Microanalyse, Moscou-Kiev (1974).

[6] Blaise, G. et Castaing, R., C.R. Hebd. Séan. Acad. Sci. 284B (1977) 449 ; J. Microsc. Spectrosc. Electron. 3 (1978) 439.

[7] Blaise, G., Material Characterization Using Ion Beams édité par J.-P. Thomas et A. Cachard (Plenum Press, LondresNew York) NATO B 28 (1978) 143.

[8] Wassmuth, H. W. et Müller, R., Surf. Sci. 46 (1974) 441.

[9] Hennequin, J.-F. et Couchouron, M., III e Colloque International de Physique et Chimie des Surfaces, Grenoble (1977).

[10] Thomas, G. E. et de Kluizenaar, E. E., Rev. Sci. Instrum. 45 (1974) 457

[11] Müller, R. et Wassmuth, H.-W., Surf. Sci. 40 (1973) 15.

[12] Dawson, P. H., Quadrupole Mass Spectrometry and its Applications (Elsevier Scientific Publishing Company, Amsterdam) 1976.
[13] Hennequin, J.-F. et Inglebert, R.-L., Int. J. Mass Spectrom. Ion Phys. 26 (1978) 131

[14] Hennequin, J.-F. et Inglebert, R.-L., Revue Phys. Appl. 14 (1979) 275.

[15] Körner, W., Phys. Status Solidi a 22 (1974) 523.

[16] Haque, C. A. et Donaldson, E. E., Rev. Sci. Instrum. 34 (1963) 409.

[17] Zandberg, E. Ya., Ionov, N. I. et Tontegode, A. Ya., Sov. Phys. Tech. Phys. 10 (1966) 1164.

[18] Scheer, M. D. et Fine, J., J. Chem. Phys. 46 (1967) 3998.

[19] Hoffert, F., Paulus, J. et Adloff, J.-P., Revue Phys. Appl. 1 (1966) 43.

[20] Pottie, R. F., Cocke, D. L. et Gingerich, K. A., Int. J. Mass Spectrom. Ion Phys. 11 (1973) 41.

[21] FeHN, U., Int. J. Mass Spectrom. Ion Phys. 21 (1976) 1.

[22] Sigmund, P., Phys. Rev. 184 (1969) 383.

[23] Andersen, H. H. et BAy, H. L., Radiat. Eff. 13 (1972) 67 ; 19 (1973) 139.

[24] Almen, O. et Bruce, G., Nucl. Instrum. Methods 11 (1961) 257.

[25] Hennequin, J.-F. et Couchouron, M., J. Microsc. Spectrosc. Electron. 3 (1978) 439.

[26] Hanszen, K.-J. et Lauer, R., Focusing of Charged Particles édité par A. Septier (Academic Press, New York-London) I (1967) 251.

[27] Inglebert, R.-L. et Hennequin, J.-F., VIIIth International Mass Spectrometry Conference, Oslo (1979); Revue Phys. Appl. (en préparation). 